\title{
Physical Conditions and Star Formation Activity in the Intragroup Medium of Stephan's Quintet ${ }^{1,2,3}$
}

\author{
C. K. Xu, N. Lu \\ Infrared Processing and Analysis Center, Jet Propulsion Laboratory, Caltech 100-22, Pasadena, CA 91125 \\ J. J. Condon \\ National Radio Astronomy Observatory ${ }^{1}, 520$ Edgemont Road, Charlottesville, VA 22903 \\ Electronic mail: jcondon@nrao.edu \\ M. Dopita \\ Research School of Astronomy \& Astrophysics, The Australian National University, Cotter Road, Weston \\ Creek ACT 2611, Australia \\ R.J. Tuffs \\ Max-Planck-Institut für Kernphysik, Postfach 103980, D69117 Heidelberg, Germany
}

\begin{abstract}
New multi-band observations of the famous compact group of galaxies Stephan's Quintet (SQ) are presented and analyzed. These include far infrared (FIR) images at $60 \mu \mathrm{m}$ and $100 \mu \mathrm{m}$ (ISOPHOT C-100 camera), radio continuum images at $1.4 \mathrm{GHz}$ (VLA B-array) and $4.86 \mathrm{GHz}$ (VLA C-array), and long-slit optical spectrographs (Palomar 200" telescope). With these new data, we aim to learn more about the X-ray/radio ridge in the middle of the intragroup medium (IGM) and the IGM starburst SQ-A, both are likely to be caused by the high speed collision $\left(\sim 900 \mathrm{~km} \mathrm{~s}^{-1}\right)$ between the intruder galaxy NGC 7318b $\left(v=5700 \mathrm{~km} \mathrm{~s}^{-1}\right)$ and the IGM $\left(v=6600 \mathrm{~km} \mathrm{~s}^{-1}\right)$. We found that the radio ridge has a steep nonthermal spectral index $(\alpha=0.93 \pm 0.13)$ and an extremely low FIR-to-radio ratio index $(q<0.59)$. Its IR emission can be explained in terms of collisional heating of dust grains by shocked gas. The minimum-energy magnetic field strength is $H_{\min } \approx 10 \mu \mathrm{G}$. The long-slit spectra of sources in the ridge have typical emission line ratios of shock-excited gas. The very broad line widths $\left(\geq 1000 \mathrm{~km} \mathrm{~s}^{-1}\right)$, and the fact that in some cases more than two velocity systems were detected along the same line of sight, provide further evidence for an ongoing collision along the ridge. The IGM starburst SQ-A has a radio spectral index $\alpha=0.8 \pm 0.3$ and a FIR-to-radio ratio index $q=2.0 \pm 0.4$, consistent with those of star forming regions. The optical spectra of two sources in this region, M1 $\left(v=6600 \mathrm{~km} \mathrm{~s}^{-1}\right)$ and M2 $\left(v=6000 \mathrm{~km} \mathrm{~s}^{-1}\right)$, have typical line ratios of HII regions. Both M1 and M2 have metallicity slightly higher than the solar value. The star formation rate (SFR) estimated from the extinction-corrected $\mathrm{H}_{\alpha}$ luminosity of SQ-A is $1.45 \mathrm{M}_{\odot} \mathrm{yr}^{-1}$, of which 1.25 $\mathrm{M}_{\odot} \mathrm{yr}^{-1}$ is due to the $v=6600 \mathrm{~km} \mathrm{~s}^{-1}$ component and $0.20 \mathrm{M}_{\odot} \mathrm{yr}^{-1}$ to the $v=6000 \mathrm{~km} \mathrm{~s}^{-1}$ component.
\end{abstract}

\footnotetext{
${ }^{1}$ The National Radio Astronomy Observatory is a facility of the National Science Foundation operated under cooperative agreement by Associated Universities, Inc.

${ }^{2}$ Based on observations made with ISO, an ESA project with instruments funded by ESA Member States and with the participation of ISAS and NASA.

${ }^{3}$ Based on observations obtained at the Hale Telescope, Palomar Observatory as part of a continuing collaboration between the California Institute of Technology, NASA/JPL, and Cornell University.
} 
Subject headings: galaxies: interactions - galaxies: intergalactic medium - galaxies: ISM galaxies: starburst - galaxies: active - infrared: galaxies - stars: formation

\section{Introduction}

There is a resurgent enthusiasm recently on the investigations of the historically famous Stephan's Quintet (hereafter SQ), a multi-galaxy system discovered by Stephan in late 19th century (Stephan 1887). As summarized in Sulentic et al. (2001), many new observational data have recently become available for this intriguing source. In particular, a few new frequency windows have been opened to astronomical observations in this space era. The X-ray observations using ROSAT (Sulentic et al. 1995; Saracco \& Ciliegi 1995; Pietsch et al. 1997; Sulentic et al. 2001) and Chandra (Trinchieri et al. 2003), infrared observations using ISO (Xu et al. 1999; Sulentic et al. 2001), and very high angular resolution optical images using HST (Gallagher et al. 2001) have revealed intriguing new features in this fascinating galaxy group. Particularly, an unusual IR source was discovered by Xu et al. (1999) in the intragroup medium (IGM) of SQ, located more than $20 \mathrm{kpc}$ away from any neighboring galaxy centers. The IR source, named Source A (hereafter SQ-A) by Xu et al. (1999), is apparently associated with a starburst triggered by the high speed collision (relative velocity $\sim 1000 \mathrm{~km} \mathrm{~s}^{-1}$ ) between the intruder galaxy NGC $7318 \mathrm{~b}$ and the IGM. It is the location in the IGM that makes the starburst so unusual. This is also the first example of an ongoing starburst triggered by collisions of such high speed, though evidence for a post-starburst was found previously by Kenney et al (1995) in the high speed colliding system NGC 4438/4439.

Another outstanding feature in SQ is the large scale shock front $(\sim 40 \mathrm{kpc})$ in the IGM between NGC 7319 and NGC 7318b, first discovered by Allen \& Hartsuiker (1973) as an radio emission ridge in the $21 \mathrm{~cm}$ Westerbork radio continuum map, and later confirmed by the VLA observations of van der Hulst \& Rots (1981) and of Williams et al. (2003). The high resolution X-ray maps of SQ (Pietsch et al. 1997; Sulentic et al. 2001; Trinchieri et al. 2003) show an X-ray emission ridge at almost exactly the same position, making it very certain that this ridge is the signature of a shock front. Given the fact that the relative velocity between the gas-rich intruder galaxy NGC 7318b and the rest of the group is $\sim 900 \mathrm{~km}$ $\mathrm{s}^{-1}$, and that there is widely spread cold HI gas in the IGM (Shostak et al. 1984; Williams et al. 2002), it is indeed expected that such a shock must be happening in the region where the cold gas associated with the intruder collides with the cold gas in the IGM.

$\mathrm{Xu}$ et al. (1999) argued strongly that the two phenomenal events, namely the ongoing IGM starburst and the large scale shock, are intimately related with each other: the starburst is very likely triggered by the same collision that triggered the shock. In this paper, we present new observations in the infrared, radio continuum, and optical spectroscopy for SQ. With these new data and data found in the literature, we aim to better constrain the physical conditions in the IGM, particularly in the shock front region and in SQ-A, and investigate further the physical mechanism linking the two events. The paper is organized as follows: after this introduction, the new observations are presented in Section 2 through Section 4. In Section 5, we study the physical conditions in the IGM. In Section 6 we investigate the triggering mechanism for the IGM starburst SQ-A. The Section 7 is devoted to a discussion, and the conclusions are presented in Section 8. We assume that the distance of SQ is $80 \mathrm{Mpc}$, and the distance of the foreground interloper NGC 7320 is $10 \mathrm{Mpc}$. 


\section{Mapping of IR emission with ISO}

\subsection{Observations}

The mapping observations of SQ were carried out on May 23, 1996 using ISOCAM and ISOPHOT on board ISO (Kessler et al. 1996). The total on-target time for the ISOCAM maps (at $15 \mu \mathrm{m}$ and $11.4 \mu \mathrm{m}$ ) is $33 \mathrm{ks}$, and for the ISOPHOT maps (at $60 \mu \mathrm{m}$ and $100 \mu \mathrm{m}$ ) is $14 \mathrm{ks}$. These maps are among the most sensitive observations ever made by ISO. The $15 \mu \mathrm{m}, 11.4 \mu \mathrm{m}$ and $60 \mu \mathrm{m}$ maps have already been published in Xu et al. (1999) and in Sulentic et al. (2001). In this paper we present the $100 \mu m$ map, which is the last product from our ISO project. In Table 1, we summarize the ISO observations.

Table 1. Summary of ISO Observations of Stephan's Quintet

\begin{tabular}{|c|c|c|c|c|c|c|c|c|c|c|}
\hline camera & $\begin{array}{c}\text { array } \\
\text { size }\end{array}$ & filter & $\begin{array}{c}\lambda \\
(\mu \mathrm{m})\end{array}$ & $\begin{array}{c}\delta \lambda \\
(\mu \mathrm{m})\end{array}$ & $\begin{array}{c}\text { detector } \\
\text { pixel }\end{array}$ & $\begin{array}{c}\text { samp. } \\
\text { step }\end{array}$ & $\begin{array}{c}\text { map } \\
\text { size }\end{array}$ & $\begin{array}{c}\text { rms } \\
(\mathrm{MJy} / \mathrm{sr})\end{array}$ & $\begin{array}{c}\text { rms } \\
\left(\mathrm{mJy} / \mathrm{beam}^{\dagger}\right)\end{array}$ & $\begin{array}{c}\text { calib. } \\
\mathrm{err} .\end{array}$ \\
\hline $\begin{array}{c}\text { ISOCAM } \\
\text {-LW }\end{array}$ & $32 \times 32$ & LW8 & 11.4 & 2.3 & $6^{\prime \prime} \times 6^{\prime \prime}$ & $6^{\prime \prime} \times 6^{\prime \prime}$ & $5^{\prime} \times 12^{\prime}$ & 0.083 & 0.22 & $\sim 20 \%$ \\
\cline { 2 - 11 } & $32 \times 32$ & LW3 & 15.0 & 6.0 & $6^{\prime \prime} \times 6^{\prime \prime}$ & $6^{\prime \prime} \times 6^{\prime \prime}$ & $5^{\prime} \times 12^{\prime}$ & 0.047 & 0.12 & $\sim 20 \%$ \\
\hline $\begin{array}{c}\text { ISOPHOT } \\
\text {-C100 }\end{array}$ & $3 \times 3$ & C60 & 60.8 & 23.9 & $45^{\prime \prime} \times 45^{\prime \prime}$ & $15^{\prime \prime} \times 15^{\prime \prime}$ & $6^{\prime} \times 13^{\prime}$ & 0.33 & 14 & $\sim 20 \%$ \\
\cline { 2 - 10 } & $3 \times 3$ & C100 & 103.5 & 43.6 & $45^{\prime \prime} \times 45^{\prime \prime}$ & $15^{\prime \prime} \times 15^{\prime \prime}$ & $6^{\prime} \times 13^{\prime}$ & 0.40 & 16 & $\sim 20 \%$ \\
\hline
\end{tabular}

$\dagger$ Beam areas are calculated assuming Gaussian beams of FWHM of $10^{\prime \prime}$ for the ISOCAM maps (under-sampled) and

FWHM of $40^{\prime \prime}$ for the ISOPHOT maps (over-sampled).

Both the $100 \mu \mathrm{m}$ and the $60 \mu \mathrm{m}$ maps were taken using the ISOPHOT C-100 camera in the observation mode P32 (oversampling mapping mode). The ISOPHOT P32 mode combines the standard spacecraft raster pointing mode (stepping in spacecraft $\mathrm{Y}$ and $\mathrm{Z}$ coordinates) with scans in $\mathrm{Y}$ made with the focal plane chopper (Tuffs \& Gabriel 2003; see also Tuffs et al. 2002a,b). At each spacecraft raster position the focal plane chopper is stepped at intervals of one third of the detector pixel pitch, resulting in sky samplings in $\mathrm{Y}$ of $\sim 15^{\prime \prime}$ for the C100 detector, which is close to the limit for Nyquist sampling of $\left(17^{\prime \prime} \times \lambda\right) / 100 \mu \mathrm{m}$. The oversampling factor of 3 was chosen for both maps (sampling step of $\sim 15^{\prime \prime}$ in both in-scan and cross-scan directions). Both maps have 16 map lines (scans) and 9 pointings per map line, covering about 13' (in scan) $\times 6^{\prime}$ (cross scan) sky area (Table 1$)$. The rational of choosing relatively long scans $\left(13^{\prime}\right.$ compared to the size of SQ of $\sim 5^{\prime}$ ) is two-folds: (1) to facilitate the correction of the transient response behavior of the Ge:Ga photoconductor detectors of ISOPHOT; (2) to enclose enough empty background sky regions surrounding the system and therefore the maps will be sensitive to any tenuous diffuse emission associated with the intragroup medium outside main bodies of member galaxies.

The ISOPHOT data were reduced using the latest version of the P32 data reduction package (Tuffs \& Gabriel 2003). This software takes the advantage of high sampling rate and high redundancy of P32 maps. The effects of detector transient were properly corrected (failure to correct for these effects in data taken in the "P32" mode can give rise to serious signal losses and distortions in the derived brightness profiles through the maps). Detailed descriptions of the software and of the standard data reduction procedure can be found in Tuffs \& Gabriel (2003). During the reduction procedure it became clear that, for the $100 \mu m$ map, three (pixel 1,4 and 5) of the nine detector pixels of the C-100 camera are rather noisy. These pixels were therefore excluded from the final map making. 
The final calibrations for both the $100 \mu \mathrm{m}$ and $60 \mu \mathrm{m}$ were determined using a tool provided by the P32 data reduction package, which compares the local background with that measured by IRAS and COBE, taking also into account the seasonal variation of the zodiacal light. As pointed out by Tuffs \& Gabriel (2003), this procedure can remove any residual systematic uncertainties remaining after the transient response correction, and yield close agreements with IRAS calibrations. At 60 and $100 \mu m$, brightnesses on the ISO maps were multiplied by factors of 1.04 and 0.81 , respectively, to bring the background level on the ISOPHOT maps into equivalence with the background estimated from COBE/DIRBE. Aperture photometry using the final $100 \mu \mathrm{m}$ and $60 \mu \mathrm{m}$ maps finds total flux densities (and rms errors) of SQ of $f_{100 \mu m}=2.22( \pm 0.03)$ Jy and $f_{60 \mu m}=1.07( \pm 0.03) \mathrm{Jy}$, in good agreement with the IRAS values of $f_{100 \mu m}=2.54 \pm 0.36 \mathrm{Jy}$ and $f_{60 \mu m}=0.88 \pm 0.09 \mathrm{Jy}$. From these comparisons, the IRAS values are consistent with the ISO values to within 20 percent. In what follows we will adopt a conservative estimate of 20 percent for the systematic calibration uncertainty of the ISO data.

\subsection{Results}

In Fig. 1a and Fig. 1b we present the images of the $100 \mu m$ and $60 \mu m$ maps, respectively. In both maps the background is quite smooth and the noise behaves normally. No obvious artifacts can be seen in the images except for near the edges. The emission in both maps is dominated by two sources, one associated with the Seyfert galaxy NGC 7319 (on the right), the other with the foreground galaxy NGC 7320. These two galaxies were marginally resolved and detected in the $60 \mu \mathrm{m}$ IRAS HIRES map (Allam et al. 1996). In other three IRAS bands $(12,25$, and $100 \mu m)$, only NGC 7319 was detected in the IRAS HIRES study of Allam et al. (1996).

In both ISOPHOT $100 \mu m$ and $60 \mu m$ maps, the source associated with NGC 7319 is slightly elongated along the scan direction $\left(\mathrm{P} . \mathrm{A} .=167^{\circ}\right)$, with the size $(\mathrm{FWHM})$ along the major axis being $48^{\prime \prime}$ and $54^{\prime \prime}$, respectively, and the minor/major axis ratio being 0.78 and 0.61 , respectively. This might indicate that, in addition to the IR emission of the Sy2 nucleus which dominates the MIR emission of NGC 7319 (Xu et al. 1999; Sulentic et al. 2001), the dust in the host galaxy may contribute significantly to the FIR emission. The $\mathrm{H}_{\alpha}$ arm on the north of the nucleus (Xu e al. 1999; see also Fig. 6 below) where massive molecular gas was also detected (Yun et al. 1997; Gao \& Xu 2000), may indeed be active star formation regions in the disk of NGC 7319 and therefore be bright in the FIR. On the other hand, it cannot be ruled out that the elongation is caused by some residual transient effects in the ISO maps, given the coincidence with the

Fig. 1.- ISOPHOT images of Stephan's Quintet. 
scan direction. Future observations with higher angular resolutions (e.g. using SIRTF-MIPS) will help to distinguish these two possibilities.

In Fig. 2a, 2b, 2c and 2d, contours of the $100 \mu m, 60 \mu m, 15 \mu m$, and $11.4 \mu m$ maps are overlaid on the $15 \mu \mathrm{m}$ image, respectively. Sources A, B, C, D, together with member galaxies of SQ, are marked on Fig. 2c (the $15 \mu \mathrm{m}$ image, see also Fig. of Xu et al. 1999). Comparisons with ISOCAM maps show that the IGM starburst SQ-A, as well as the main body of the galaxy pair NGC 7318a/b are clearly detected in the longer wavelength ISOPHOT maps. Another weaker IGM starburst discovered in the ISOCAM $15 \mu m$ map (Xu et al. 1999), Source B (hereafter SQ-B), also appears to be detected in both the $100 \mu m$ and the $60 \mu m$ maps. However, its ISOPHOT flux densities are highly uncertain (at about a factor of 2 level) because SQ-B is located on a background plateau due to a more extended, at same time rather structured, low-emission region and therefore it is difficult to subtract the background accurately.

It is always difficult to measure flux densities for individual sources in a crowded field such as SQ. This problem becomes even more severe in the ISOPHOT maps where the angular resolutions are poor $\left(\sim 40^{\prime \prime}\right)$. Indeed, the ISOPHOT flux densities of the weaker sources such as SQ-A and NGC 7318a/b had to be measured on the residual maps after subtracting the two bright sources NGC 7319 and NGC 7320. In this procedure, the two bright sources are approximated by two 2-dimensional Gaussians in each of the ISOPHOT maps, and are then subtracted from the map. In Fig. 3a and Fig. 3b, we present the contours of the residual $100 \mu \mathrm{m}$ map and $60 \mu \mathrm{m}$ map, respectively. Given the surface brightness distributions of NGC 7319 and of NGC 7320 in the higher resolution ISOCAM maps, the 2-dimensional Gaussian model should be a good approximation. Nevertheless, the ISOPHOT flux densities of SQ-A and NGC 7318a/b have large uncertainties due to the sensitive dependence on the subtraction of brighter sources.

In Table 2, we list the IR flux densities of the individual sources measured from the ISO maps. Except for the flux densities presented with brackets denoting the high uncertainties (see discussion above), the errors of the measurements are on the order of $20 \%$, mostly due to the adopted (rather conservative) calibration uncertainty (see Section 2.1). The errors of those flux densities with brackets can easily be $50 \%$ or more. The coordinates of the galaxies are taken from NED, while the positions of the ISOCAM sources (Source A through D) are measured from the ISOCAM $15 \mu \mathrm{m}$ image, with uncertainties on the order of $2^{\prime \prime}$.

In the $100 \mu \mathrm{m}$ map, there is clear evidence for extended emission (at $\sim 1 \mathrm{MJy} / \mathrm{sr}$ level) in the periphery of SQ, with a broad wing passing through SQ-B. Since much of this region is not in the same scans that pass though the bright sources, the diffuse emission is unlikely to be due to any residual transient effects. An estimate of the integrated flux densities of the diffuse emission is given by subtracting the sums of the flux densities of the discrete sources listed in Table 2 (respectively 0.68 and 1.32 Jy at 60 and $100 \mu m$ ), from the total flux densities of SQ (equal to 1.07 and 2.22 Jy at the same two wavelengths). This yields values of 0.39 and $0.90 \mathrm{Jy}$ for the diffuse component at 60 and $100 \mu \mathrm{m}$, respectively, which is about 40 percent of the total. This is one of a few examples where diffuse dust emission is detected outside galaxies (see Tuffs \& Popescu 2002 for a review on this topic). The diffuse emission around SQ-B is likely to be powered by young stars formed in the same tidal tail where SQ-B is located. Braine et al. (2001) and Lisenfeld et al. (2002) detected massive molecular gas in this region. In other places, it might be powered either by stars stripped from galaxies during previous close encounters between SQ members (Moles et al. 1997; Sulentic et al. 2001), or by the diffuse X-ray emitting gas (Trinchieri et al. 2003). This will be a very interesting problem for future, more sensitive IR observations.

The early type galaxy NGC 7317, detected by ISOCAM at both $15 \mu m$ and $11.4 \mu m$, was not detected by ISOPHOT, nor were ISOCAM sources Source C (a star in the north of SQ) and Source D (a background 
galaxy in the south of SQ).

Table 2. ISO Flux Densities of Sources in SQ Field

\begin{tabular}{|c|c|c|c|c|c|c|c|}
\hline Name & $\begin{array}{l}\text { R.A. } \\
\text { (J2000) }\end{array}$ & $\begin{array}{l}\text { Dec. } \\
(\mathrm{J} 2000)\end{array}$ & $\begin{array}{l}f_{11.4 \mu m} \\
(\mathrm{mJy})\end{array}$ & $\begin{array}{l}f_{15 \mu m} \\
(\mathrm{mJy})\end{array}$ & $\begin{array}{l}f_{60 \mu m} \\
(\mathrm{mJy})\end{array}$ & $\begin{array}{l}f_{100 \mu m} \\
\text { (mJy) }\end{array}$ & note \\
\hline NGC 7317 & 223552.0 & +335641 & 1.6 & 2.2 & & & E galaxy \\
\hline Shock \& NGC 7318a/b & 223557.6 & +335757 & 23.9 & 19.2 & $(76)$ & $(230)$ & shock \& g. pair \\
\hline NGC 7319 & 223603.5 & +335833 & 54.5 & 76.3 & 275 & 350 & Sy2 galaxy \\
\hline NGC 7320 & 223603.5 & +335654 & 46.7 & 27.3 & 260 & 609 & f.g. galaxy \\
\hline SQ-A & 223558.7 & +335850 & 10.9 & 10.9 & $(60)$ & $(106)$ & IGM starburst \\
\hline SQ-B & 223610.2 & +335721 & 0.9 & 1.7 & $(13)$ & $(21)$ & IGM starburst \\
\hline SQ-C & 223602.7 & +335956 & 3.3 & 0.6 & & & star \\
\hline SQ-D & 223600.1 & +335552 & $(0.2)$ & 0.7 & & & b.g. galaxy \\
\hline
\end{tabular}

$\dagger$ These 3 regions are grouped together because they can hardly be separated from each other in the ISOPHOT maps (Fig. 3).

$\ddagger$ The ISOPHOT flux densities of SQ-A were measured in the region outlined by the northwest peak in the residual $60 \mu m$ map (Fig. $3 \mathrm{~b}$ ).

\section{Radio Continuum Observations with the VLA}

\subsection{Observations and Data Reduction}

There have been several interferometric radio continuum observations of SQ in the literature (Allen \& Hartsuiker 1973; van der Hulst \& Rots 1981; Williams et al. 2002; Xanthopoulos et al. 2002). With new high sensitivity, high angular resolution VLA imaging observations in two bands (1.40 and 4.86 GHz), we aim to constrain the spectral indices of individual sources. We also try to detect the polarization, particularly in the shock front region, in an attempt to constrain the magnetic field.

In order to image the radio continuum brightness, polarization, and spectral index distributions of SQ, we observed SQ with the VLA at $1.4 \mathrm{GHz}$ in B configuration for 5 hrs on 1999 November 15 and at $4.86 \mathrm{GHz}$ in $\mathrm{C}$ configuration for $8 \mathrm{hrs}$ on 2000 April 27. During both observations, the source 3C 48 $\left(S_{1.40 \mathrm{GHz}}=16.19 \mathrm{Jy}, S_{4.86 \mathrm{GHz}}=5.52 \mathrm{Jy}\right.$, Baars et al. 1977) was used to calibrate the fringe amplitudes to the updated VLA flux-density scale. The polarization position angles were determined from 3C 138, and the nearby source $\mathrm{J} 2236+284$ was used as the phase calibrator.

Preliminary total-intensity (Stokes I) images were made and cleaned by the AIPS task IMAGR. 
To maximize surface-brightness sensitivity and in the same time minimize the synthesized sidelobes, we used tapered uniform weighting with Gaussian amplitude tapering to 30\% at 25,000 wavelengths and truncated at 45,000 wavelengths. This strong taper yields a large synthesized beam area having the high surface-brightness sensitivity of natural weighting. The nearly uniform weighting below 25,000 wavelengths reduces the dirty-beam sidelobes that natural weighting would produce because the central part of the VLA synthetic aperture would be "over-illuminated." Then the clean components from these images were subtracted from the $(u, v)$ data. All residual visibilities having amplitudes much larger than the rms noise were flagged, and the clean components were added back to the edited data set. This procedure removes low-level interference and other problems that cause individual visibilities to disagree significantly with the data set as a whole. New images of 1024 pixels $\times 1^{\prime \prime}$ pixel $^{-1}$ on a side were made, cleaned, and restored with 6!"0 FWHM circular Gaussian beams, and the final images were corrected for primary-beam attenuation. The rms noise levels are $\sigma \approx 25 \mu \mathrm{Jy}_{\text {beam }}{ }^{-1}$ at $1.40 \mathrm{GHz}$ and $\sigma \approx 17 \mu \mathrm{Jy}_{\text {beam }}{ }^{-1}$ at $4.86 \mathrm{GHz}$. Matching Stokes Q and $\mathrm{U}$ images were also made, and they have somewhat lower noise levels. No linearly polarized emission brighter than $50 \mu \mathrm{Jy}_{\text {beam }^{-1}}$ was found in any component of SQ at either 1.40 or $4.86 \mathrm{GHz}$.

\subsection{Radio Continuum Images}

In Fig. 4 we present the total-intensity contour maps of the $1.40 \mathrm{GHz}$ and $4.86 \mathrm{GHz}$ images, both overlaid on an R-band CCD image (Xu et al. 1999).

The following radio sources are visible in these maps and their fitted parameters are listed in Table 3:

(1) There is a strong steep-spectrum source whose centroid at J2000 $\alpha=22^{\mathrm{h}} 36^{\mathrm{m}} 03 \stackrel{\mathrm{s}}{5} 55, \delta=+33^{\circ} 58^{\prime} 32^{\mathrm{s}} .6$ overlaps the optical nucleus of the Seyfert 2 galaxy NGC 7319 at $\alpha=22^{\mathrm{h}} 36^{\mathrm{m}} 03.56, \delta=+33^{\circ} 58^{\prime} 33^{\prime \prime} .2$. We fit this source with elliptical Gaussians and deconvolved the 6 !. $^{\prime}$ restoring beams to obtain Gaussian approximations to the source FWHM major axis, minor axis, and position angle at both frequencies: $4.5 \times 1 " .6$ in $\mathrm{PA}=29^{\circ} \mathrm{E}$ of $\mathrm{N}$ in the $1.40 \mathrm{GHz}$ map, $4 . .5 \times 1 " .5$ in $\mathrm{PA}=27^{\circ}$ in the $4.86 \mathrm{GHz}$ map. These sizes and orientations are reliable even though the deconvolved source size is smaller than the beam because the signal-to-noise ratio of this source is so high in our images. They are consistent with the linear triple structure found in higher-resolution VLA images at 3.6, 6, and $20 \mathrm{~cm}$ (Aoki et al. 1999). The precise agreement of our $1.40 \mathrm{GHz}$ integrated flux density with theirs (28.5 mJy in both cases) indicates that there can be very little extended emission surrounding the triple. The orientation of the radio triple is nearly parallel to the $\mathrm{H}_{\alpha^{-}}[\mathrm{N} \mathrm{II}]$ emission-line region visible in Fig. 2 of $\mathrm{Xu}$ et al. (1999). This phenomenon is common in Seyfert galaxies and suggests that the radio jets are brightening the line-emitting gas by compression.

(2) There is a weak radio source in NGC 7318a, the early type galaxy in the binary system NGC 7318a/b. This radio source is unresolved (FWHM $<4^{\prime \prime}$ ) in our images and centered on $\alpha=22^{\mathrm{h}} 35^{\mathrm{m}} 56^{\mathrm{s}} .72$, $\delta=+33^{\circ} 57^{\prime} 56^{\prime \prime} 0$, precisely coincident with the optical nucleus at $\alpha=22^{\mathrm{h}} 35^{\mathrm{m}} 56^{\mathrm{s}} .74$, $\delta=+33^{\circ} 57^{\prime} 56^{\prime \prime} 3$ (Klemola et al. 1987). The source has been detected previously by van der Hulst \& Rots (1981) and by Williams et al. (2002) at 1.4 Ghz. Our measurement of the $S_{1.40 G H z}(0.95 \pm 0.05$ mJy) is slightly ( 2 sigma) lower than that of Williams et al. $(1.4 \pm 0.2)$.

(3) There is a radio ridge at $\alpha \approx 22^{\mathrm{h}} 36^{\mathrm{m}} 00^{\mathrm{s}}$, which extends north-south from $\delta \approx+33^{\circ} 57^{\prime} 10^{\prime \prime}$ to $\delta \approx+33^{\circ} 58^{\prime} 40^{\prime \prime}$, then bends west and terminates near $\alpha=22^{\mathrm{h}} 35^{\mathrm{m}} 56^{\mathrm{s}}, \delta=+33^{\circ} 59^{\prime} 15^{\prime}$ (Allen \& Hartsuiker, 1973; van der Hulst \& Rots, 1981; Williams et al. 2002). The radio ridge is coextensive 
with the X-ray ridge (Pietsch et al. 1997), the ridge of the high-redshift $\left(6600 \mathrm{~km} \mathrm{~s}^{-1}\right)$ component of the $\mathrm{H}_{\alpha^{-}}[\mathrm{N} \mathrm{II}]$ emission (Xu et al. 1999), and the FIR emission shown in Fig. 3. With a very faint optical continuum counterpart, the ridge appears to delineate a shock front containing relativistic electrons, magnetic fields, hot thermal electrons, ionized gas, and cool dust. The $1.4 \mathrm{GHz}$ flux density, $S_{1.40 G \mathrm{~Hz}}=34.7 \pm 3.5 \mathrm{mJy}$, is about $30 \%$ lower than the result of Williams et al. (2002) obtained using their lower resolution image which is $S_{1.40 \mathrm{GHz}}=48 \pm 7 \mathrm{mJy}$, indicating that our high-resolution B-array image may be missing about 13 mJy of flux from the ridge.

(4) The relatively weak and diffuse radio peak near $\alpha=22^{\mathrm{h}} 35^{\prime} 58^{\mathrm{s}} .8, \delta=+33^{\circ} 58^{\prime} 50^{\prime \prime}$ overlaps the IGM starburst SQ-A just north of the bend in the ridge. Its 1.40 and $4.86 \mathrm{GHz}$ flux densities were estimated by fitting Gaussians to SQ-A above base planes fitted to the surrounding ridge emission.

(5) The binary radio source (called SQ-R in Table 3 ), with the stronger component at $\alpha=22^{\mathrm{h}} 36^{\mathrm{m}} 00$ s. 01 , $\delta=+33^{\circ} 59^{\prime} 12^{\prime \prime} .3$ and the weaker component at $\alpha=22^{\mathrm{h}} 35^{\mathrm{m}} 59^{\mathrm{s}} .9, \delta=+33^{\circ} 59^{\prime} 02^{\prime \prime}$, is almost certainly cosmologically distant background source seen in projection behind SQ (van der Hulst \& Rots, 1981; Williams et al. 2002). It has no optical counterpart brighter than $29 \mathrm{mag}^{\operatorname{arcsec}^{-2}}$ (Williams et al. 2002), nor any IR counterpart in the ISO images. The $1.40 \mathrm{GHz}$ flux density is in excellent agreement with Williams et al. (2002).

(6) The weak IGM starburst SQ-B (near the eastern boundary of the images) is marginally detected in both $1.40 \mathrm{GHz}$ and $4.86 \mathrm{GHz}$ maps.

The flux densities of these sources are listed in Table 3. Their total $1.40 \mathrm{GHz}$ flux is 76.5 mJy, somewhat smaller than the $S_{1.4}=93 \pm 4 \mathrm{mJy}$ from the D-configuration NVSS image, so our B-configuration image may be missing up to $16.5 \mathrm{mJy}$ of diffuse flux. A comparison with the total flux density $\left(S_{1.4}=96 \pm 15\right.$ mJy) from the C and D configurations image of Williams et al. (2002) leads to a similar conclusion. 
Fig. 2.- Contour maps of the IR emission (background subtracted) at $100 \mu m, 60 \mu m, 15 \mu m$, and $11.4 \mu \mathrm{m}$ overlaid on the $15 \mu \mathrm{m}$ image. The contour levels for the $100 \mu \mathrm{m}$ map are $[2,2.8,4,5.6,8,11.2,16] \times 0.4$ $\mathrm{MJy} / \mathrm{sr}$; for the $60 \mu \mathrm{m}$ map they are $[2,2.8,4,5.6,8,11.2,16,22.4] \times 0.33 \mathrm{MJy} / \mathrm{sr}$; for the $15 \mu \mathrm{m}$ map they are $[2,2.8,4,5.6,8,11.2,16,22.4,32] \times 0.047 \mathrm{MJy} / \mathrm{sr}$; for the $11.4 \mu \mathrm{m}$ map they are $[2,2.8,4,5.6,8,11.2,16] \times 0.083$ $\mathrm{MJy} / \mathrm{sr}$. Sources detected in the $15 \mu \mathrm{m}$ map are labeled according to Xu et al. (1999).

Fig. 3.- Contour maps of the $100 \mu m$ and $60 \mu m$ emission after the subtraction of NGC 7319 and NGC 7320, overlaid on the $15 \mu \mathrm{m}$ image. The contour levels for the $100 \mu \mathrm{m}$ map are $[2,2.8,4,5.6] \times 0.4 \mathrm{MJy} / \mathrm{sr}$; for the $60 \mu \mathrm{m}$ map they are $[2,2.8,4,5.6] \times 0.33 \mathrm{MJy} / \mathrm{sr}$.

Fig. 4.- Left: Contours of the radio continuum at $1.40 \mathrm{GHz}$ (VLA B-array) overlaid on an R-band CCD image. The lowest contour is $90 \mu \mathrm{Jy}$ beam $^{-1}$ and the spacing $=2$ in ratio. Right: Contours of the radio continuum at $4.86 \mathrm{GHz}$ (VLA C-array) overlaid on the same $\mathrm{R}$-band image. The lowest contour is $50 \mu \mathrm{Jy}_{\text {beam }}{ }^{-1}$ and the spacing $=2$ in ratio. For both radio maps the FWHM of the synthesized beam is $6{ }^{\prime \prime} 0$. 
Table 3. Radio Continuum Properties of Sources in SQ Field

\begin{tabular}{|l|l|l|l|l|c|c|}
\hline Name & $\begin{array}{l}\text { R.A. } \\
(\mathrm{J} 2000)\end{array}$ & $\begin{array}{l}\text { Dec. } \\
(\mathrm{J} 2000)\end{array}$ & $\begin{array}{l}S_{1.40 \mathrm{GHz}} \\
\text { mJy }\end{array}$ & $\begin{array}{l}S_{4.86 \mathrm{GHz}} \\
\text { mJy }\end{array}$ & $\begin{array}{l}\text { spect. index } \\
(\alpha)\end{array}$ & $\begin{array}{c}\text { FIR/radio index } \\
(\mathrm{q})\end{array}$ \\
\hline Ridge & 223559.8 & +335800 & $34.7 \pm 3.5$ & $10.9 \pm 1.1$ & $0.93 \pm 0.13$ & $<0.59^{\dagger}$ \\
\hline NGC 7318a & 223556.72 & +335756.0 & $0.95 \pm 0.05$ & $0.44 \pm 0.03$ & $0.62 \pm 0.07$ & \\
\hline NGC 7319 & 223603.55 & +335832.6 & $28.5 \pm 0.5$ & $9.7 \pm 0.3$ & $0.87 \pm 0.03$ & $1.08 \pm 0.10$ \\
\hline SQ-A & 223558.8 & +335850 & $0.8 \pm 0.2$ & $0.3 \pm 0.1$ & $0.8 \pm 0.3$ & $2.0 \pm 0.4$ \\
\hline SQ-B & 223610.2 & +335721 & $0.6 \pm 0.2$ & $0.2 \pm 0.1$ & $0.7 \pm 0.4$ & $1.5 \pm 0.7$ \\
\hline SQ-R & 223600.01 & +335912.3 & $10.3 \pm 1.0$ & $3.7 \pm 0.4$ & $0.85 \pm 0.12$ & $\cdots$ \\
\hline
\end{tabular}

$\dagger$ The $q$ (upperlimit) is calculated using the ISOPHOT flux densities of a region including also NGC 7318a/b (Table 3).

In Fig. 5 we present the contour map of the spectral index $\alpha=\log \left(S_{1.4} / S_{4.86}\right) / \log (4.86 / 1.4)$ overlaid on the ISOCAM $15 \mu \mathrm{m}$ map. It should be noted that there is little effect of the missing flux on the spectral index analysis because $(1)$ the $(u, v)$-plane coverages and synthetic beams of the two images are very similar and therefore about same fraction of flux is missed in the two bands, and (2) the flux missed is mostly in the diffuse emission that contributes little to the flux ratios of the relatively high surface brightness regions where the spectral index analysis is confined. Both the $1.40 \mathrm{GHz}$ and the $4.86 \mathrm{GHz}$ maps were smoothed to a common Gaussian beam of FWHM $=10^{\prime \prime}$, the beam size of the ISOCAM map, before the calculation of the spectral index. Only pixels with $\mathrm{s} / \sigma>2$ in both maps were included in the calculation. Both the background binary source and the strong source associated with the Seyfert 2 galaxy NGC 7319 have fairly normal spectral indices $\alpha \sim 0.8$, and the radio source associated with NGC 7318a has a slightly flatter spectral index $\alpha \sim 0.6$. A large part of the radio ridge associated with the shock front has $0.7<\alpha<1.1$, typical for the synchrotron emission from relativistic electrons, while a small part of the ridge immediately south of SQ-A shows steep $(\alpha \sim 1.2)$ spectrum. It is interesting to note that, albeit not being plotted by the spectral index contours in Fig. 5 because of low $\mathrm{s} / \sigma$, this steep spectrum appears to extend to the northwest of SQ-A where diffuse X-ray (Trinchieri et al. 2003) and $\mathrm{H}_{\alpha}$ (Fig. 6) emission was detected. Since a steep-spectrum radio emission is usually associated with aging CR electrons, its distribution may provide constraints to the dynamic history of the shock.

Fig. 5.- Contours of radio spectral index $(\alpha)$ overlaid on ISOCAM $15 \mu m$ image. The spatial resolution is $10^{\prime \prime}$ (FWHM). Only regions having $s / \sigma \geq 2$ in both radio maps are included in the calculation of $\alpha$. The contour levels are $\alpha=0.5,0.7,0.9$ and 1.1 . 


\subsection{Comparison with IR Maps}

For normal galaxies there is a strong correlation between the IR and the radio continuum emission, not only for the total fluxes in these two bands (Helou et al. 1985; De Jong et al. 1985; Popescu et al. 2002) but also for the local emission within galaxies, for example in the LMC (Xu et al. 1992) and in M31 (Hoernes et al. 1998). The very tight correlation is generally explained by the common dependence of the two emissions on the star-formation activity (see Condon 1992, and Völk \& Xu 1994 for reviews).

However, in the case of SQ, the IR maps (Fig. 2's) and the radio continuum maps (Fig. 4) look very different. Also the ratio between the total FIR flux and the total radio continuum flux of SQ is significantly different from the typical value for normal galaxies: The ratio is often expressed by the $q$ parameter (Helou et al. 1985) which will be called the FIR-to-radio ratio index in this paper:

$$
q \equiv \log \left(\frac{F I R}{3.75 \times 10^{12} \mathrm{~W} \mathrm{~m}^{-2}}\right)-\log \left(\frac{S_{1.40 \mathrm{GHz}}}{\mathrm{W} \mathrm{m}^{-2} \mathrm{~Hz}^{-1}}\right)
$$

where

$$
F I R=1.26 \times 10^{-14}\left(\frac{2.58 f_{60 \mu \mathrm{m}}+f_{100 \mu \mathrm{m}}}{\mathrm{Jy}}\right) .
$$

For SQ $\left(S_{1.40 \mathrm{GHz}}=93 \pm 10 \mathrm{mJy}, f_{60 \mu \mathrm{m}}=1.07 \pm 0.21 \mathrm{Jy}\right.$ and $\left.f_{100 \mu \mathrm{m}}=2.22 \pm 0.51 \mathrm{Jy}\right), q=1.23 \pm 0.27$, significantly lower than the mean for normal star-forming galaxies, which is $\langle q\rangle=2.3 \pm 0.2$ (Helou et al. 1985). The $q$ values for individual sources in SQ are listed in Table 3.

Noticeably, the upperlimit of the $q(<0.59)$ of the radio ridge indicates that it is more than a factor of 50 too radio-loud compared to normal star-forming galaxies. This is because the ridge is not in a galaxy dominated by ongoing star formation. Rather, most of the radio emission is from relativistic electrons accelerated by the large-scale shock, which is in turn created by the collision between the intruder and the IGM (van der Hulst \& Rots 1981; Pietsch et al. 1997). When developing a theory for the break-down of the FIR/radio correlation in clustered galaxies, Völk \& Xu (1994) considered a scenario bearing many similarities with what is actually happening in SQ (though SQ is a group, not a cluster). They envisaged a high speed collision between a galaxy and a clump of cold intracluster medium, the latter being stripped gas (e.g. by ram pressure) from a late-type galaxy. The collision produces a large amount of cosmic-ray electrons which in turn radiate strongly in the radio continuum, while little FIR emission is produced.

Another reason for the low $q$ value of SQ is the radio emission from the AGN in the Seyfert 2 galaxy NGC 7319, which accounts for about one-third of the emission in SQ in both the IR and radio wavebands. NGC 7319 has a $q=1.10$ (Table 3), low compared to the mean of normal star-forming galaxies but in the range of those of AGN's (Condon \& Broderick 1988). The physical mechanisms responsible for the relation between the radio and the FIR emissions in AGNs are completely different from those in normal star-forming galaxies (Condon \& Broderick 1988).

Given the relatively large uncertainty, the $q$ value of SQ-A $(q=2.0 \pm 0.4)$ is consistent with normal star formation. For SQ-B, another starburst in the IGM of SQ, $q=1.5 \pm 0.7$. This is substantially lower than the mean, though only at $\sim 1 \sigma$ significance level because of the large error bar. SQ-B has large uncertainties in both the FIR and the radio continuum flux densities, mainly because it is located on a background plateau due to a more extended, at same time rather structured, low-emission region. This makes not only the the background subtraction difficult but also the boundaries of the emission associated with this source highly uncertain. 


\section{Long-Slit Optical Spectroscopy}

\subsection{Observations}

Long-slit optical spectroscopic observations were made on September 21, 1999 (UT) using the Double

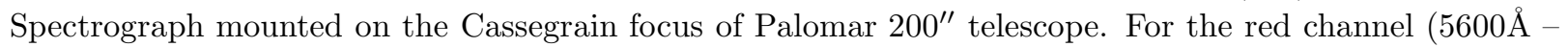
$8000 \AA), 316$ lines $/ \mathrm{mm}$ grating mode $(102 \AA / \mathrm{mm})$ was chosen, and for the blue channel $(3700 \AA-5400 \AA)$, 600 lines $/ \mathrm{mm}$ grating mode $(71 \AA / \mathrm{mm})$ was used. Two $1024 \times 1024$ CCD detectors, one for each channel, have pixel size of $24 \mu \mathrm{m}$. The slit is $128^{\prime \prime}$ long and $2^{\prime \prime}$ wide. The spectral dispersions for the red and blue spectra are $2.4 \AA /$ pix and $1.7 \AA$ /pix, respectively. Measured from the bright sky lines, the FWHM spectral resolutions are $7.5 \AA$ for the red channel and $4.6 \AA$ for the blue. These correspond to velocity resolutions of $343 \mathrm{~km} \mathrm{~s}^{-1}$ at $\mathrm{H}_{\alpha}$ and $283 \mathrm{~km} \mathrm{~s}^{-1}$ at $\mathrm{H}_{\beta}$, respectively.

Observations were carried out along two slits: Slit M and Slit N (Fig. 6). Slit M is centered at (J2000) $22 \mathrm{~h} 35 \mathrm{~m} 58.7 \mathrm{~s}+33^{\circ} 58^{\prime} 50^{\prime \prime}$, with a position angle of $128 \mathrm{deg}$. Slit $\mathrm{N}$ is centered at (J2000) $22 \mathrm{~h} 35 \mathrm{~m} 59.8 \mathrm{~s}$ $+33^{\circ} 58^{\prime} 00^{\prime \prime}$, with a position angle of $176 \mathrm{deg}$. Three exposures, each lasting for 20 minutes, were carried out for each slit orientation. The spectra derived from these individual exposures were coadded for each slit orientation during the data reduction which was done using IRAF developed by National Optical Astronomy Observatories.

The standard neon and hollow cathode lamp spectra were taken before and after each source observation for the purpose of wavelength calibration. For each red or blue spectrum, about 20 to 25 bright emission lines with accurately known wavelengths were used to determine a wavelength solution with an accuracy of better than $0.2 \AA$. The flux calibration was done via observations of the standard stars $\mathrm{BD}+28^{\circ} 4211$ and G191B2B from (Oke, 1990) in a 4"-wide slit. The night was not strictly photometric, with some thin cirrus clouds and a variable seeing between $1.2^{\prime \prime}$ to $1.5^{\prime \prime}$. While the relative flux calibration across a spectrum should be quite good, the absolute flux calibration is less certain, probably not much better than $30 \%$.

Fig. 6.- Positions of Slit M and Slit N. Each is $128^{\prime \prime}$ long and 2" wide. The labels "1", "2", "3" and "4" along Slit M and "a", "b", "c", "d" and "e" along Slit N mark the approximate positions of the regions

where emission lines are detected (Table 4). The background, taken from Fig. 2 of Xu et al. (1999), is the $\mathrm{K}^{\prime}(2.1 \mu \mathrm{m})$ image overlaid by contours of the $\mathrm{H}_{\alpha}-[\mathrm{NII}]$ emission. 
Fig. 7.- Spectrographs of the IGM star formation regions and of the shock front in Stephan's Quintet (see Fig. 6 for the locations of Slit M and Slit N). The approximate positions are marked for redshifted

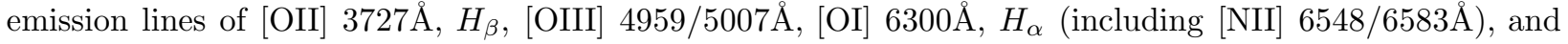
[SII] $6717 / 6731 \AA$. Emission regions along Slit $\mathrm{M}$ and Slit $\mathrm{N}$ are labeled (near $H_{\alpha} /[\mathrm{NII}]$ lines) in the same way as in Fig. 6.

Fig. 8.- Blue $(3700 \AA-5400 \AA)$ and $\operatorname{red}(5600 \AA-8000 \AA)$ spectra of 4 sources along Slit M.

Fig. 9.- Blue $(3700 \AA-5400 \AA)$ and red $(5600 \AA-8000 \AA)$ spectra of 5 sources along Slit N. 
Table 4. Spectroscopic Sources along the Two Slits

\begin{tabular}{lllc}
\hline Source ID & R.A. (J2000) & Dec. (J2000) & aperture \\
\hline & & & \\
M1 & $22^{\mathrm{h}} 35^{\mathrm{m}} 59.18^{\mathrm{s}}$ & $33^{\circ} 58^{\prime} 45.3^{\prime \prime}$ & $9^{\prime \prime} \times 2^{\prime \prime}$ \\
$\mathrm{M} 2$ & $22^{\mathrm{h}} 35^{\mathrm{m}} 58.11^{\mathrm{s}}$ & $33^{\circ} 58^{\prime} 55.6^{\prime \prime}$ & $15^{\prime \prime} \times 2^{\prime \prime}$ \\
$\mathrm{M} 3$ & $22^{\mathrm{h}} 36^{\mathrm{m}} 01.90^{\mathrm{s}}$ & $33^{\circ} 58^{\prime} 18.8^{\prime \prime}$ & $18^{\prime \prime} \times 2^{\prime \prime}$ \\
$\mathrm{M} 4$ & $22^{\mathrm{h}} 35^{\mathrm{m}} 56.54^{\mathrm{s}}$ & $33^{\circ} 59^{\prime} 10.9^{\prime \prime}$ & $8^{\prime \prime} \times 2^{\prime \prime}$ \\
& & & \\
$\mathrm{Na}$ & $22^{\mathrm{h}} 35^{\mathrm{m}} 59.77^{\mathrm{s}}$ & $33^{\circ} 58^{\prime} 01.8^{\prime \prime}$ & $14^{\prime \prime} \times 2^{\prime \prime}$ \\
$\mathrm{Nb}$ & $22^{\mathrm{h}} 35^{\mathrm{m}} 59.91^{\mathrm{s}}$ & $33^{\circ} 57^{\prime} 36.8^{\prime \prime}$ & $8^{\prime \prime} \times 2^{\prime \prime}$ \\
$\mathrm{Nc}$ & $22^{\mathrm{h}} 35^{\mathrm{m}} 59.62^{\mathrm{s}}$ & $33^{\circ} 58^{\prime} 26.2^{\prime \prime}$ & $8^{\prime \prime} \times 2^{\prime \prime}$ \\
$\mathrm{Nd}$ & $22^{\mathrm{h}} 35^{\mathrm{m}} 59.50^{\mathrm{s}}$ & $33^{\circ} 58^{\prime} 47.9^{\prime \prime}$ & $8^{\prime \prime} \times 2^{\prime \prime}$ \\
$\mathrm{Ne}$ & $22^{\mathrm{h}} 36^{\mathrm{m}} 00.02^{\mathrm{s}}$ & $33^{\circ} 57^{\prime} 17.4^{\prime \prime}$ & $15^{\prime \prime} \times 2^{\prime \prime}$ \\
& & & \\
\hline
\end{tabular}

In Fig. 7, we present the four spectrographs (two slits, two channels). Along Slit M and Slit N, significant signals were detected around 4 positions (M1, M2, M3, M4) and 5 positions ( $\mathrm{Na}, \mathrm{Nb}, \mathrm{Nc}, \mathrm{Nd}$, $\mathrm{Ne}$ ), respectively. They are listed in Table 4. The approximate positions of these sources are also marked in Fig. 6 and Fig. 7. For each source, we have defined an aperture size, given in the last column of Table 4. This aperture was used to extract a 1-d spectrum. The resulting blue and red spectra of these sources are plotted in Fig. 8 for Slit M and Fig. 9 for Slit N.

\subsection{Emission Lines}

In Table 5 we list the following measured quantities for the detected lines:

(1) the corresponding redshift in $\mathrm{km} \mathrm{s}^{-1}$;

(2) velocity dispersion (in $\mathrm{km} \mathrm{s}^{-1}$ );

(3) line flux in $10^{-15} \mathrm{erg} \mathrm{s}^{-1} \mathrm{~cm}^{-2}$. 
Table 5. Emission lines

\begin{tabular}{|c|c|c|c|c|c|c|c|c|c|c|}
\hline & \multicolumn{2}{|c|}{ M1 } & \multirow[t]{2}{*}{ M2 } & \multirow[t]{2}{*}{ M3 } & \multirow[t]{2}{*}{ M4 } & \multirow[t]{2}{*}{$\mathrm{Na}$} & \multirow[t]{2}{*}{$\mathrm{Nb}$} & \multirow[t]{2}{*}{$\mathrm{Nc}$} & \multirow[t]{2}{*}{$\mathrm{Nd}$} & \multirow[t]{2}{*}{$\mathrm{Ne}$} \\
\hline & comp. 1 & comp. 2 & & & & & & & & \\
\hline \multicolumn{11}{|l|}{$[\mathrm{SII}] \lambda 6731$} \\
\hline $\mathrm{V}\left(\mathrm{km} \mathrm{s}^{-1}\right)$ & 6686 & & 6017 & & & 6197 & 5756 & 6102 & 6693 & 6246 \\
\hline$\delta \mathrm{V}\left(\mathrm{km} \mathrm{s}^{-1}\right)$ & 381 & & 368 & & & 1010 & 1115 & 1341 & 297 & 499 \\
\hline flux $^{\dagger}$ & 0.94 & & 0.89 & & & $2.05^{\ddagger}$ & $1.40^{\ddagger}$ & $1.68^{\ddagger}$ & 0.18 & 0.62 \\
\hline \multicolumn{11}{|l|}{ [SII] $\lambda 6717$} \\
\hline $\mathrm{V}\left(\mathrm{km} \mathrm{s}^{-1}\right)$ & 6700 & 5987 & 6030 & & 6253 & 6164 & 5713 & 6020 & 6697 & 6221 \\
\hline$\delta \mathrm{V}\left(\mathrm{km} \mathrm{s}^{-1}\right)$ & 366 & 346 & 375 & & 405 & 611 & 316 & 348 & 619 & 550 \\
\hline flux $^{\dagger}$ & 1.29 & 0.16 & 1.20 & & 0.11 & $1.85^{\ddagger}$ & $0.50^{\ddagger}$ & $0.22^{\ddagger}$ & 0.40 & 1.11 \\
\hline \multicolumn{11}{|l|}{ [NII] $\lambda 6583$} \\
\hline $\mathrm{V}\left(\mathrm{km} \mathrm{s}^{-1}\right)$ & 6745 & & 5970 & 6471 & & 5889 & 5588 & 5927 & 6574 & 6251 \\
\hline$\delta \mathrm{V}\left(\mathrm{km} \mathrm{s}^{-1}\right)$ & 382 & & 429 & 513 & & 1024 & 1110 & 703 & 573 & 515 \\
\hline flux ${ }^{\dagger}$ & 1.63 & & 2.54 & 1.14 & & $8.09^{\ddagger}$ & $4.60^{\ddagger}$ & $3.60^{\ddagger}$ & 0.62 & 1.18 \\
\hline \multicolumn{11}{|l|}{$\mathrm{H}_{\alpha}$} \\
\hline $\mathrm{V}\left(\mathrm{km} \mathrm{s}^{-1}\right)$ & 6673 & & 5988 & 6445 & 6125 & 6152 & 5722 & 6060 & 6677 & 6249 \\
\hline$\delta \mathrm{V}\left(\mathrm{km} \mathrm{s}^{-1}\right)$ & 364 & & 357 & 433 & 342 & 495 & 403 & 430 & 576 & 558 \\
\hline flux $^{\dagger}$ & 10.0 & & 5.64 & 1.27 & 0.24 & $2.79^{\ddagger}$ & $1.48^{\ddagger}$ & $1.78^{\ddagger}$ & 2.87 & 3.76 \\
\hline \multicolumn{11}{|l|}{ [NII] $\lambda 6548$} \\
\hline $\mathrm{V}\left(\mathrm{km} \mathrm{s}^{-1}\right)$ & 6689 & & 6001 & 6414 & & 6156 & 5695 & 6142 & 6682 & 6251 \\
\hline$\delta \mathrm{V}\left(\mathrm{km} \mathrm{s}^{-1}\right)$ & 354 & & 309 & 426 & & 596 & 807 & 288 & 345 & 364 \\
\hline flux $^{\dagger}$ & 0.91 & & 0.58 & 0.37 & & $0.80^{\ddagger}$ & $0.56^{\ddagger}$ & $0.16^{\ddagger}$ & 0.58 & 0.41 \\
\hline \multicolumn{11}{|l|}{$[\mathrm{OI}] \lambda 6300$} \\
\hline $\mathrm{V}\left(\mathrm{km} \mathrm{s}^{-1}\right)$ & 6761 & & & & & 6392 & 6317 & 6622 & 6676 & 6268 \\
\hline$\delta \mathrm{V}\left(\mathrm{km} \mathrm{s}^{-1}\right)$ & 587 & & & & & 1134 & 1038 & 1268 & 557 & 623 \\
\hline flux $^{\dagger}$ & 0.41 & & & & & 1.90 & 0.78 & 0.92 & 0.22 & 0.79 \\
\hline
\end{tabular}

${ }^{\dagger}$ Flux of the line emission in $10^{-15} \mathrm{erg} \mathrm{s}^{-1} \mathrm{~cm}^{-2}$.

‡ Severely blended. 
Table 5. Continue

\begin{tabular}{|c|c|c|c|c|c|c|c|c|c|}
\hline & \multicolumn{2}{|c|}{ M1 } & \multirow[t]{2}{*}{ M2 } & \multirow[t]{2}{*}{ M3 } & \multirow[t]{2}{*}{$\mathrm{Na}$} & \multirow[t]{2}{*}{$\mathrm{Nb}$} & \multirow[t]{2}{*}{$\mathrm{Nc}$} & \multirow[t]{2}{*}{$\mathrm{Nd}$} & \multirow[t]{2}{*}{$\mathrm{Ne}$} \\
\hline & comp. 1 & comp. 2 & & & & & & & \\
\hline \multicolumn{10}{|l|}{ He I $\lambda 5876$} \\
\hline $\mathrm{V}\left(\mathrm{km} \mathrm{s}^{-1}\right)$ & 6688 & & 6024 & & & & & & \\
\hline$\delta \mathrm{V}\left(\mathrm{km} \mathrm{s}^{-1}\right)$ & 383 & & 219 & & & & & & \\
\hline flux $^{\dagger}$ & 0.35 & & 0.19 & & & & & & \\
\hline \multicolumn{10}{|l|}{$[\mathrm{OIII}] \lambda 5007$} \\
\hline $\mathrm{V}\left(\mathrm{km} \mathrm{s}^{-1}\right)$ & 6530 & 5812 & 5812 & & 6830 & 5512 & & 6591 & \\
\hline$\delta \mathrm{V}\left(\mathrm{km} \mathrm{s}^{-1}\right)$ & 303 & 269 & 264 & & 298 & 206 & & 276 & \\
\hline flux ${ }^{\dagger}$ & 3.59 & 0.43 & 1.42 & & 0.31 & 0.22 & & 0.66 & \\
\hline \multicolumn{10}{|l|}{ [OIII] $\lambda 4959$} \\
\hline $\mathrm{V}\left(\mathrm{km} \mathrm{s}^{-1}\right)$ & 6534 & & 5868 & & & & & & \\
\hline$\delta \mathrm{V}\left(\mathrm{km} \mathrm{s}^{-1}\right)$ & 287 & & 316 & & & & & & \\
\hline flux $^{\dagger}$ & 1.05 & & 0.38 & & & & & & \\
\hline \multicolumn{10}{|l|}{$\mathrm{H}_{\beta}$} \\
\hline $\mathrm{V}\left(\mathrm{km} \mathrm{s}^{-1}\right)$ & 6665 & & 5986 & & & 5678 & & 6789 & 6233 \\
\hline$\delta \mathrm{V}\left(\mathrm{km} \mathrm{s}^{-1}\right)$ & 285 & & 342 & & & 316 & & 422 & 339 \\
\hline flux $^{\dagger}$ & 1.45 & & 1.49 & & & 0.37 & & 0.44 & 0.83 \\
\hline \multicolumn{10}{|l|}{$\mathrm{H}_{\gamma}$} \\
\hline $\mathrm{V}\left(\mathrm{km} \mathrm{s}^{-1}\right)$ & 6635 & & 6014 & & & & & & \\
\hline$\delta \mathrm{V}\left(\mathrm{km} \mathrm{s}^{-1}\right)$ & 279 & & 335 & & & & & & \\
\hline flux $^{\dagger}$ & 0.39 & & 0.67 & & & & & & \\
\hline \multicolumn{10}{|l|}{ [OII] $\lambda 3727$} \\
\hline $\mathrm{V}\left(\mathrm{km} \mathrm{s}^{-1}\right)$ & 6600 & 5876 & & & 6117 & 5796 & & 6600 & 6198 \\
\hline$\delta \mathrm{V}\left(\mathrm{km} \mathrm{s}^{-1}\right)$ & 451 & 355 & & & 526 & 283 & & 937 & 487 \\
\hline flux $^{\dagger}$ & 1.82 & 1.08 & & & 2.31 & 0.82 & & 1.48 & 2.34 \\
\hline
\end{tabular}

${ }^{\dagger}$ Flux of the line emission in $10^{-15} \mathrm{erg} \mathrm{s}^{-1} \mathrm{~cm}^{-2}$.

In Fig. 10 we present close-up plots of the following lines: $[0 \mathrm{II}] \lambda 3727, \mathrm{H}_{\beta},[\mathrm{OIII}] \lambda 4959 / \lambda 5007,[\mathrm{OI}] \lambda 6300$, $\mathrm{H}_{\alpha} /[\mathrm{NII}] \lambda 6548 / \lambda 6563,[\mathrm{SII}] \lambda 6716 / \lambda 6731$.

\subsection{Notes for Individual Sources}

M1: The brightest source, near the center of the IGM starburst associated with the MIR source SQ-A (Xu et al. 1999). The line emission is predominantly from the $6600 \mathrm{~km} \mathrm{~s}^{-1}$ component (comp. 1 in Table 5). The most conspicuous evidence for the second component (comp. 2 in Table 5), centered at $\sim 5900 \mathrm{~km} \mathrm{~s}^{-1}$, is the second peak of the $[\mathrm{OII}] \lambda 3727$ line. This second component also shows up in 
the $[\mathrm{OIII}] \lambda 5007$ line and the $[\mathrm{SII}] \lambda 6716$ line.

M2: The second brightest source, also associated with the MIR source SQ-A. However, different from M1, the line emission of this source is from the $6000 \mathrm{~km} \mathrm{~s}^{-1}$ component. The emission from the $6600 \mathrm{~km}$ $\mathrm{s}^{-1}$ component is not detected here. This is in good agreement with Moles et al. (1998).

M3: This is a relatively faint source, located within the $\mathrm{H}_{\alpha}$ bridge linking the shock front and the Seyfert-2 nucleus of NGC 7319. Only one component centered at $\sim 6450 \mathrm{~km} \mathrm{~s}^{-1}$ is detected. The marginal detection of the $[\mathrm{SII}] \lambda 6716$ line at $6600 \mathrm{~km} \mathrm{~s}^{-1}$ is deemed unreliable because of the large errors associated with the skyline subtraction. The high $I([N I I] \lambda 6583) / I\left(H_{\alpha}\right)$ ratio $(0.90)$ suggests that the $\mathrm{H}_{\alpha}$ bridge is either a cooling flow (Heckman et al. 1989), or gas bow-shocked by an AGN jet (Aoki et al. (1999).

M4: Another weak source, associated with a diffuse emission region in the periphery of the IGM starburst. The $I([S I I] \lambda 7317) / I\left(H_{\alpha}\right)$ ratio indicates that the emission is shock excited rather than radiatively excited (Dopita \& Sutherland 1995). Apparently it is associated with the Source 5 in the Chandra image (Fig 1 of Trinchieri et al. 2003).

Na: Located near the center of the shock front. Three velocity systems $\left(6800,6100\right.$ and $\left.6400 \mathrm{~km} \mathrm{~s}^{-1}\right)$ are detected in the emission lines, with the $6100 \mathrm{~km} \mathrm{~s}^{-1}$ component dominating the $\mathrm{H}_{\alpha}$, [NII], [SII] and [OII] lines, and the $6800 \mathrm{~km} \mathrm{~s}^{-1}$ component dominating the high excitation [OIII] $\lambda 5007$ line. The very broad $[O I] \lambda 6300$ line $\left(\delta V=1134 \mathrm{~km} \mathrm{~s}^{-1}\right)$, which is the only line showing the $6400 \mathrm{~km} \mathrm{~s}^{-1}$ component, may well be due to the blending of the same line in the 6100 and $6800 \mathrm{~km} \mathrm{~s}^{-1}$ systems. The relatively large velocity offset of the $[N I I] \lambda 6583$ line from $6100 \mathrm{~km} \mathrm{~s}^{-1}$, and its large velocity dispersion $\left(\delta V=1024 \mathrm{~km} \mathrm{~s}^{-1}\right)$ may also be attributed to the contamination of the $\mathrm{H}_{\alpha}$ emission of the $6800 \mathrm{~km} \mathrm{~s}^{-1}$ component. From the line width of the [OIII] $\lambda 5007$ line and of the [OII] $\lambda 3727$ line, the $6100 \mathrm{~km} \mathrm{~s}^{-1}$ component has substantially higher velocity dispersion $\left(\delta V=526 \mathrm{~km} \mathrm{~s}^{-1}\right)$ than the $6800 \mathrm{~km} \mathrm{~s}^{-1}$ component $\left(\delta V=298 \mathrm{~km} \mathrm{~s}^{-1}\right)$.

$\mathrm{Nb}$ : Located at the intersection between the shock front and a tidal tail of the intruder NGC $7318 \mathrm{~b}$. Two velocity systems $\left(6300\right.$ and $\left.5700 \mathrm{~km} \mathrm{~s}^{-1}\right)$ are detected in the emission lines. The very broad $[O I] \lambda 6300,[N I I] \lambda 6583$ and $[S I I] \lambda 6731$ lines suggest possible blending with lines in another velocity system of $\mathrm{V} \sim 6600 \mathrm{~km} \mathrm{~s}^{-1}$. The line width of [OIII] $\lambda 5007, \mathrm{H}_{\beta}$ and [OII] $\lambda 3727$ indicates that, without blending, the velocity dispersion of the $5700 \mathrm{~km} \mathrm{~s}^{-1}$ component is $\sim 300 \mathrm{~km} \mathrm{~s}^{-1}$ (close to the velocity resolution).

Fig. 10.- Close-up plots of the following lines: [0II $] \lambda 3727, \mathrm{H}_{\beta}, \quad[\mathrm{OIII}] \lambda 4959 / \lambda 5007, \quad[\mathrm{OI}] \lambda 6300$, $\mathrm{H}_{\alpha} /[\mathrm{NII}] \lambda 6548 / \lambda 6563,[\mathrm{SII}] \lambda 6716 / \lambda 6731$. 
Nc: Located immediately north of $\mathrm{Na}$, this is another source associated with the shock front. Two velocity systems $\left(6100 \mathrm{~km} \mathrm{~s}^{-1}\right.$ and $\left.6600 \mathrm{~km} \mathrm{~s}^{-1}\right)$ are detected. As in $\mathrm{Na}$, the $6100 \mathrm{~km} \mathrm{~s}^{-1}$ component dominates the $\mathrm{H}_{\alpha}$, [NII], and [SII] lines. But, unlike in Na, the central velocity of the $[O I] \lambda 6300$ line $\left(6622 \mathrm{~km} \mathrm{~s}^{-1}\right.$ ) indicates that the high velocity component dominates this line emission, though the broad line width $\left(\delta V=1268 \mathrm{~km} \mathrm{~s}^{-1}\right)$ suggests contribution from the low velocity component.

Nd: Associated to the same starburst region as M1, but off-set by $\sim 3^{\prime \prime}$ toward the east. Only one component associated with the IGM $\left(6600 \mathrm{~km} \mathrm{~s}^{-1}\right)$ is detected. The [SII] lines are affected by sky-line subtraction, therefore with relatively large uncertainties.

Ne: At the southern end of the shock front, likely to be associated with the post-shock gas. Only one component is detected, with the central velocity of $\sim 6250 \mathrm{~km} \mathrm{~s}^{-1}$.

\section{Physical Conditions in the IGM of SQ}

\subsection{In the Ionized Gas}

\subsubsection{IGM Starburst ( $S Q-A)$ Region}

Four sources (M1, M2, M4 and Nd) in Table 5 are in this region. M1 and M2 are close to the core of the IGM starburst, and M4 and Nd are in the periphery. As indicated in previous HI, CO and Fabry-Pèrot observations, there are two velocity systems with recession velocities of $\sim 6600 \mathrm{~km} \mathrm{~s}^{-1}$ and $\sim 6000 \mathrm{~km} / \mathrm{sec}$, respectively, in this region. The main component of M1 (comp. 1) and Nd belong to the $6600 \mathrm{~km} \mathrm{~s}^{-1}$ system, while the second component of M1 and M2 belong to the $6000 \mathrm{~km} \mathrm{~s}^{-1}$ system. M4, with a velocity of $\sim 6200 \mathrm{~km} \mathrm{~s}^{-1}$, is perhaps associated with the hot X-ray gas (see Section 4.3) and has no counterpart in the neutral gas.

We have detected most of the diagnostic lines in both M1 and M2 with relatively high signal-to-noise ratios. Both sources show typical HII-region line ratios. In Table 6 we list the physical parameters derived from the line ratios:

Table 6. Physical Conditions in SQ-A

\begin{tabular}{lccccc}
\hline $\begin{array}{l}(1) \\
\text { source }\end{array}$ & $\begin{array}{c}(2) \\
12+\log [O / H]\end{array}$ & $\begin{array}{c}(3) \\
n_{e} \\
\left(\mathrm{~cm}^{-3}\right)\end{array}$ & $\begin{array}{c}T_{\text {ion }} \\
(\mathrm{K})\end{array}$ & $\begin{array}{c}(5) \\
\mathrm{Q}\end{array}$ & $\begin{array}{c}(6) \\
\mathrm{A}\left(\mathrm{H}_{\alpha}\right) \\
(\mathrm{mag})\end{array}$ \\
\hline $\mathrm{M} 1$ & $8.76 \pm 0.06$ & $<100$ & $\sim 40000$ & $\sim 10^{8}$ & 2.0 \\
$\mathrm{M} 2$ & $8.95 \pm 0.09$ & $<100$ & $\sim 40000$ & $\sim 10^{7}$ & 0.64 \\
\hline
\end{tabular}

Notes:

column (2): Metal abundance derived using the model of Kewley \& Dopita (2002). To be compared with the Solar value $12+\log [O / H]=8.69 \pm 0.05$ (Allende Prieto et al. 2001)

column (3): Density derived from $I([S I I] \lambda 6717) / I([S I I] \lambda 6731)$. 
column (4): Ionization temperature derived, using the model of Evans \& Dopita (1985), from two extinction-insensitive line ratios: $I([O I I I] \lambda 5007) / I\left(H_{\beta} \lambda 4861\right)$ and $I([N I I] \lambda 6584) / I\left(H_{\alpha} \lambda 6563\right)$.

column (5): Ionization parameter derived, using the model of Evans \& Dopita (1985), from two extinction-insensitive line ratios: $I([O I I I] \lambda 5007) / I\left(H_{\beta} \lambda 4861\right)$ and $I([N I I] \lambda 6584) / I\left(H_{\alpha} \lambda 6563\right)$.

column (6): $\mathrm{H}_{\alpha}$ extinction derived from Balmer decrement, assuming the Case $\mathrm{B}$ intrinsic line ratio $I\left(H_{\alpha} \lambda 6563\right) / I\left(H_{\beta} \lambda 4861\right)=2.86$ and average Galactic interstellar extinction curve (Savage and Mathis 1979).

It appears that both cold gas systems, as revealed by the data of M1 and M2, have the metal abundance slightly above the solar value. They both have density $<100 \mathrm{~cm}^{-3}$ and $T_{i o n} \sim 40000 \mathrm{~K}$. These are in the range of typical HII regions. The ionization parameter of M2 is an order of magnitude lower than that of M1, indicating the former has a lower density and/or larger distance from the ionizing stars. This is consistent with the more extended morphology and much lower extinction of M2 compared to M1. The extinction derived from the Balmer decrement of $\mathrm{M} 1$ is $\mathrm{A}\left(\mathrm{H}_{\alpha}\right)=2 \mathrm{mag}$, consistent with its rather red continuum color (Sulentic et al. 2001) and the very compact morphology of the $6600 \mathrm{~km} \mathrm{~s}^{-1} \mathrm{HI}$ gas (Williams et al. 2002).

\subsubsection{Shock-front Region}

$\mathrm{Na}, \mathrm{Nb}, \mathrm{Nc}$ and $\mathrm{Ne}$ are in the shock front region. They all have $I([O I] \lambda 6300) / I\left(H_{\alpha}+[N I I]\right)>0.1$ and $I([S I I] \lambda 6717,6713) / I\left(H_{\alpha}+[N I I]\right)>0.25$, therefore are dominantly shock excited (Dopita \& Sutherland 1995). The kinematics of the ionized gas in these sources is rather complex, but consistent with previous results from the HI and Fabry-Pérot observations (Sulentic et al. 2001). In Na and Nc, which are north of NGC 7318b, most of the emission lines are dominantly due to the low velocity $\left(6000 \mathrm{~km} \mathrm{~s}^{-1}\right)$ system. Indeed, the HI observations (Williams et al. 2002) detected only the $6000 \mathrm{~km} \mathrm{~s}^{-1}$ component in this region. The high velocity system $\left(6600 \mathrm{~km} \mathrm{~s}^{-1}\right)$, with which the low velocity HI gas is colliding, shows up only in [OI] $\lambda 6300$, and [OIII] $\lambda 5007$ lines.

The fact that the $6600 \mathrm{~km} \mathrm{~s}^{-1}$ component is undetected in $\mathrm{HI}$ in shock front suggests that most of it has been processed by the shock and, consequently, been converted to hot gas or ionized gas (Sulentic et al. 2001). In the framework of the models of Dopita \& Sutherland (1995), this means that the $6600 \mathrm{~km}$ $\mathrm{s}^{-1}$ component does not have the precursor HII region in front of the shock front. This may be one of the reasons why $6600 \mathrm{~km} \mathrm{~s}^{-1}$ component is relatively faint in these regions because, without the precursor, its Balmer lines are about a factor of 2.3 fainter (Dopita \& Sutherland 1995).

Because of the severe blending among [SII] and [NII] lines, it is difficult to derive the density and other physical parameters using line ratios. Nevertheless, given the close relation between the ionized gas in the shock front and in SQ-A, it is likely that the $6600 \mathrm{~km} \mathrm{~s}^{-1}$ and $6000 \mathrm{~km} \mathrm{~s}^{-1}$ components have the metal abundance of M1 and M2, respectively. From the extended morphology of the $\mathrm{H}_{\alpha}$ emission (Xu et al. 1999) and of the $6000 \mathrm{~km} \mathrm{~s}^{-1} \mathrm{HI}$ gas in the shock front, one can safely infer a rather low gas density (much lower than that in M1), possibly in the range of $n_{e} \sim 0.01-0.1 \mathrm{~cm}^{-3}$ as derived by Trinchieri et al. (2003) from the X-ray and $\mathrm{HI}$ data. For a gas temperature of $5.810^{6} \mathrm{~K}$ (Trinchieri et al. 2003) and a head-on collision $\left(\phi=90^{\circ}\right)$ between the clouds, the shock velocity is $v_{\text {shock }} \sim 460 \mathrm{~km} \mathrm{~s}^{-1}$ (Eq. 1 
of Trinchieri et al. 2003). This is very close to the shock velocity derived from the line-of-sight relative velocity (400 $\mathrm{km} \mathrm{s}^{-1}=4 / 3 \times 600 / 2 \mathrm{~km} \mathrm{~s}^{-1}$ ), confirming that the direction of the collision is nearly parallel to the line-of-sight (Sulentic et al. 2001).

The non-detection of the $H_{\beta}$ emission in Na corresponds to an upperlimit of $\mathrm{I}\left(\mathrm{H}_{\beta}\right)<0.310^{-15}$ $\mathrm{erg} \mathrm{s}^{-1} \mathrm{~cm}^{-2}$. Assuming an intrinsic $\mathrm{I}\left(\mathrm{H}_{\alpha}\right) / \mathrm{I}\left(\mathrm{H}_{\beta}\right)=3$ (Dopita \& Sutherland 1995), a very high extinction $\left(\mathrm{E}\left(\mathrm{H}_{\beta}-\mathrm{H}_{\alpha}\right)>1.2\right)$ is hinted at when comparing the $H_{\beta}$ upperlimit with the $H_{\alpha}$ flux reported in Table 5. Indeed, substantial IR emission is detected around Na region (Fig. 2).

$\mathrm{Nb}$ and $\mathrm{Ne}$ are in the south of NGC 7318b. Nb is very close (within a few arcsec) to the two 'unusual emission line regions' (U1 and U2) observed by Ohyama et al. (1998). Nb, U1 and U2 all show lines consistent with shock excitation models. However, the velocities of U1 and U2 are $6560 \mathrm{~km} \mathrm{~s}^{-1}$ and 6729 $\mathrm{km} \mathrm{s}^{-1}$ (Ohyama et al. 1998), while the emission lines (except for the [OI] line) in $\mathrm{Nb}$ are dominated by the $5700 \mathrm{~km} \mathrm{~s}^{-1}$ component. The spectroscopic observations of Gallagher et al. (2001) for a region near $\mathrm{Nb}$ also confirm that the co-existence of both the $5700 \mathrm{~km} \mathrm{~s}^{-1}$ and $6600 \mathrm{~km} \mathrm{~s}^{-1}$ emission line systems. It is likely that these emission line regions associated with different gas systems are ionized by the UV light generated by the same shock that is induced by their collision.

Located near the south end of the shock front, $\mathrm{Ne}$ looks different from $\mathrm{Na}, \mathrm{Nb}$ and $\mathrm{Nc}$, in the sense that only one velocity system is detected, and none of the emission lines shows the very broad $(1000+\mathrm{km}$ $\left.\mathrm{s}^{-1}\right)$ line width commonly found in the other three sources. In addition, the velocity of $\mathrm{Ne}(\mathrm{V}=6250 \mathrm{~km}$ $\mathrm{s}^{-1}$ ) is inconsistent with either $5700 \mathrm{~km} \mathrm{~s}^{-1}$ or $6600 \mathrm{~km} \mathrm{~s}^{-1}$, the two velocity systems found in the HI gas around this position. It is very likely that this emission region is due to post-shock gas which is now cooling through emission lines. Its [SII] line ratio (1.79 with an uncertainty of $20 \%$ ) and Balmer decrement are consistent with a low density gas of moderate extinction $\left(\mathrm{E}\left(\mathrm{H}_{\beta}-\mathrm{H}_{\alpha}\right)=0.45\right)$.

\subsection{Magnetic Field in the Shock-front Region}

The ridge radio source, which is associated with the shock front, emits $L_{1.40 \mathrm{GHz}} \approx 3 \times 10^{22} \mathrm{~W} \mathrm{~Hz}^{-1}$ from a volume extending about $90^{\prime \prime} \times 15^{\prime \prime}\left(\sim 10^{23} \mathrm{~cm} \times 2 \times 10^{22} \mathrm{~cm}\right)$ on the sky plane. To estimate the minimum-energy (equipartition) magnetic field strength, we assume that its depth along the line of sight is also $\sim 2 \times 10^{22} \mathrm{~cm}$, the filling factor is unity, and the relativistic ion/electron energy ratio is $k \approx 40$, the value for cosmic rays in our Galaxy. The minimum-energy field is $H_{\min } \approx 10 \mu \mathrm{G}$. The corresponding energy density of relativistic particles plus magnetic fields is $U_{\min } \approx 1.0 \times 10^{-11} \mathrm{erg} \mathrm{cm}^{-3}$. This is significantly lower than the IGM thermal energy density implied by the X-ray emission, so the radio source is dynamically insignificant. Its observed morphology is but a passive reflection of the $\mathrm{X}$-ray and $[\mathrm{N}$ II] distributions. The lack of detectable polarization in the ridge suggests that the ridge magnetic fields may be disordered, as expected if their configuration is determined by external forces. Both "beam depolarization" and Faraday rotation may have caused the reduced polarization.

The ridge radio luminosity exceeds by at least a factor of ten the radio luminosities of all star-forming regions in SQ (NGC 7318a, SQ-A, SQ-B, plus any possible non-AGN emission from NGC 7319). If it were produced by an intracluster starburst as proposed by van der Hulst \& Rots (1981), the expected star-formation rate would be about $30 M_{\odot} \mathrm{yr}^{-1}$ and the expected free-free emission at $1.4 \mathrm{GHz}$ would be about $10 \%$ of the total (Condon 1992). The optical continuum from such a luminous starburst is not seen, and our spectroscopic data and the $H_{\alpha}$ luminosity of the ridge rule out such a luminous thermal radio component. Rather, we believe that the high ridge luminosity supports the argument that shocks 
accelerated the radiating electrons, although some of the seed electrons might have been stripped from one or more normal SQ galaxies in the past.

\subsection{Dust Emission in the Shock-front Region}

The morphological resemblance between the ridge of FIR emission in the residual map (Fig. 3) and the diffuse ridge of radio synchrotron emission (Fig. 4) is striking. Thus, the FIR emission in the shock region might also be associated with the passage of the shock front. If a sufficient fraction of the refractory elements in the colliding clouds is in the solid state, the plasma immediately downstream of the shock will primarily cool by inelastic collisions of electrons and ions on dust grains. Dwek \& Werner (1981) showed that for the conditions of the interstellar medium in the solar neighborhood, this cooling mechanism dominates over cooling via inelastic collisions between electron and ions if the gas temperature immediately downstream of the shock exceeds about $10^{6} \mathrm{~K}$. For the X-ray ridge in SQ, Trinchieri et al. (2003) found a temperature of $0.5 \mathrm{KeV}\left(5.810^{6} \mathrm{~K}\right)$.

Another necessary condition for the dust emission to dominate the cooling of a shock is that dust grains can survive the sputtering during the cooling. For gas temperatures between $10^{6}$ and $10^{9} \mathrm{~K}$, Draine \& Salpeter (1979) give the sputtering time scale for a spherical grain of radius $a$ embedded in a plasma of hydrogen density $n_{\mathrm{H}}$ as:

$$
t_{\mathrm{sput}} \sim 10^{6}\left[\frac{a}{\mu m}\right]\left[\frac{n_{\mathrm{H}}}{\mathrm{cm}^{-3}}\right]^{-1} \mathrm{yr}
$$

For $n_{\mathrm{H}}=0.027 \mathrm{~cm}^{-3}$ (hot gas, Trinchieri et al. 2003) and $a=0.1 \mu \mathrm{m}, t_{\mathrm{sput}}$ is $3.7 \times 10^{6} \mathrm{yr}$. This can be compared with the gas cooling time scale $t_{\text {cool }}$ due to collisions with grains. For $a=0.1 \mu \mathrm{m}$, a gas temperature of $5.810^{6} \mathrm{~K}, n_{\mathrm{H}}=0.027 \mathrm{~cm}^{-3}$, and a dust-to-gas mass ratio of $0.006, t_{\text {cool }}$ is $\sim 2.1 \times 10^{6} \mathrm{yr}$ (Dwek \& Werner 1981). Thus, if the dust-to-gas mass ratio in the intergalactic medium of SQ is of the same order as that for the ISM in the Milky Way, the sputtering time scale will be comparable to the cooling time scale, so a significant fraction of the dust grains will survive and cool the gas. As noticed by Trinchieri et al. 2003), the distribution of the hot gas is likely to be clumpy and the actual density can be significantly higher than the mean $\left(n_{\mathrm{H}}=0.027 \mathrm{~cm}^{-3}\right)$. However, since $t_{\text {cool }}$ and $t_{\text {sput }}$ have very similar dependencies on $n_{\mathrm{H}}$ and $a$, the ratio $t_{\text {sput }} / t_{\text {cool }}$ should not vary much with these quantities.

The cooling mechanism due to dust emission is rather efficient (Dwek and Werner 1981). For sufficiently fast shocks it can tap almost all the kinetic energy flux flowing through the shock, which, following Dopita \& Sutherland (1996), is:

$$
L_{\mathrm{T}}=2.1 \times 10^{42}\left[\frac{v_{\text {shock }}}{460 \mathrm{~km} \mathrm{~s}^{-1}}\right]^{3.0}\left[\frac{n_{\mathrm{O}}}{0.01 \mathrm{~cm}^{-3}}\right]\left[\frac{A}{100 \mathrm{kpc}^{-2}}\right] \mathrm{erg} \mathrm{s}^{-1} \mathrm{~cm}^{-2}
$$

where $v_{\text {shock }}\left(\sim 460 \mathrm{~km} \mathrm{~s}^{-1}\right)$ is the shock velocity, $A$ is the area of the shock and $n_{o}$ the upstream number density (for a strong shock, $n_{o} \sim n_{\mathrm{H}} / 4$ ). For the case of the colliding HI clouds in SQ, $n_{\mathrm{o}}$ and A can be estimated to be $\sim 0.01 \mathrm{~cm}^{-3}$ and $\sim 100 \mathrm{kpc}^{2}$, respectively. These values are obtained from the typical HI column density of $310^{20} \mathrm{~cm}^{-3}$ (see Fig. 5 of Sulentic et al. 2001) and by assuming the line of sight depth of the shock to be equal to the $10 \mathrm{kpc}$ extent of the radio ridge seen in the plane of the sky.

The resulting value of $2.1 \times 10^{42} \mathrm{erg} \mathrm{s}^{-1}$ for $L_{T}$ from Eq. 4 is to be compared with the observed FIR luminosity of the shock region. In Table 2 this region is bound together with the binary NGC $7318 \mathrm{~b}$ and 
NGC 7318a, with a total $f_{60 \mu m}=76 \mathrm{mJy}$ and $f_{100 \mu m}=230 \mathrm{mJy}$. The higher resolution $15 \mu \mathrm{m}$ and $11.4 \mu \mathrm{m}$ maps suggest that roughly $\sim 30 \%$ of the FIR emission is due to the shock front, the rest is contributed by the binaries (the two nuclei plus the giant star formation region in the south of NGC 7318b, see Fig. 2). We calculate the FIR luminosity of the shock region by scaling the predicted form of the SED (Fig. 11) to a value of $69 \mathrm{mJy}$ at $100 \mu \mathrm{m}$ (estimated as 30 percent of the flux density of the combined $100 \mu \mathrm{m}$ emission of $230 \mathrm{mJy}$ from the shock and N7318a/b), and integrating over frequency. Assuming the distance of $80 \mathrm{Mpc}$, this yields a total dust luminosity $L_{\text {dust }}=1.910^{42} \mathrm{erg} \mathrm{s}^{-1}$, comparable to the $L_{\mathrm{T}}$ of $2.1 \times 10^{42} \mathrm{erg} \mathrm{s}^{-1}$ derived from Eq. 4. It should also be noted that $L_{\text {dust }}$ is about an order of magnitude higher than that of the X-ray luminosity of the ridge: $L_{\mathrm{X}} \sim 1.510^{41} \mathrm{erg} \mathrm{s}^{-1} \mathrm{~cm}^{-2}$ (Trinchieri e al. 2003), supporting the argument that the dust emission is the dominant cooling mechanism for the shock.

In the cooling time scale of $2.1 \times 10^{6} \mathrm{yr}$, a shock of velocity $460 \mathrm{~km} \mathrm{~s}^{-1}$ will move a distance of 1.0 $\mathrm{kpc}$, or 2.5 arcsec at the distance of SQ. Therefore, the dust emission should trace the shock structure very closely, as predicted by Popescu et al. (2000) for the case of accretion shocks in clusters of galaxies. In the MIR maps (Fig. 2) where the shock front can be discerned from other sources, it is indeed unresolved perpendicular to the axis of the ridge. The predicted SED (Fig. 11) from shock heated dust has a flux density ratio $f_{100 \mu m} / f_{60 \mu m}$ of 3.0 , very close to the observed color ratio of the combined dust emission of the shock and NGC 7318a/b (Table 2), which is mostly due to photon heated dust. This highlights the point made by Popescu et al. (2000), in the context of FIR emission from the intracluster medium when viewed from cosmological large distances, that the intergalactic and galactic FIR emission components will be difficult to distinguish on the basis of color, despite the different dust heating mechanisms involved.

We conclude that the observed FIR emission in the shock front region can be accounted for in terms of collisional heating of the grains by the plasma immediately downstream of the shock, provided that the refractory elements in the (pre-shock) IGM of SQ are mainly in the solid state, and the dust abundance in the HI clouds is at least as much as that in the interstellar medium of the Milky Way.

Fig. 11.- The model SED of collisionally heated dust emission in the SQ shock front region. It has been scaled to a flux density of 69 mJy at a wavelength of 100 micron. In the model (Popescu et al. 2000), the spherical grains in the size range 0.001 to $0.25 \mu \mathrm{m}$ are stochastically heated in a plasma of solar abundances with $n_{\mathrm{H}}=0.027 \mathrm{~cm}^{-3}$. The ion and electron temperatures were each taken to be $5.810^{6} \mathrm{~K}$. The grains were taken to be astronomical silicate and graphite with relative abundances taken from Draine \& Lee (1984), and with a distribution in number density over size $a$ proportional to $a^{-2.5}$. This size distribution is flatter than the interstellar size distribution to take into account the effects of sputtering, as discussed in Popescu et al. (2000). The predicted SED peaks at around $120 \mu \mathrm{m}$. 


\section{SQ-A: Star Formation Rate and Triggering Mechanism}

\subsection{Star Formation Rate}

From the $\mathrm{H}_{\alpha}$ luminosity (uncorrected for the extinction) and the $15 \mu \mathrm{m}$ luminosity of SQ-A, Xu et al. (1999) found that the star formation rate is $0.66 \mathrm{M}_{\odot} \mathrm{yr}^{-1}\left(\mathrm{H}_{\alpha}\right)$ or $0.81 \mathrm{M}_{\odot} \mathrm{yr}^{-1}(15 \mu m)$. With the new spectroscopic and FIR data, we can now better constrain these estimates.

First, we exploit the new spectroscopic information to improve the determination of the $\mathrm{H}_{\alpha}$ fluxes using the two narrow band $\left(\mathrm{H}_{\alpha}+[\mathrm{NII}]\right.$ emission) images, one primarily samples the $6600 \mathrm{~km} \mathrm{~s}^{-1}$ component and the other the $5700 / 6000 \mathrm{~km} \mathrm{~s}^{-1}$ component (Xu et al. 1999). Assuming the $\mathrm{H}_{\alpha}$-to-[NII] line ratios and the extinction corrections of the $6600 \mathrm{~km} \mathrm{~s}^{-1}$ component are the same as those of M1, and those of the $6000 \mathrm{~km} \mathrm{~s}^{-1}$ component are the same as those of $\mathrm{M} 2$, we obtain the $\mathrm{H}_{\alpha}$ fluxes (before and after extinction correction) and the $\mathrm{H}_{\alpha}$ luminosities of the two components in SQ-A (Table 7). The total uncorrected $\mathrm{H}_{\alpha}$ flux, $1.3010^{-13} \mathrm{erg} \mathrm{s}^{-1} \mathrm{~cm}^{-2}$, is in very good agreement with the result of Xu et al. (1999) of $1.2710^{-13} \mathrm{erg}$ $\mathrm{s}^{-1} \mathrm{~cm}^{-2}$. The star formation rate (SFR) can be derived from the $\mathrm{H}_{\alpha}$ luminosities using the STARBURST code (Leitherer et al. 1999) with the following parameters: continuous star formation, Salpeter IMF, $M_{\text {low }}=1 M_{\odot}, M_{\text {up }}=100 M_{\odot}$, age of the starburst: $10 \mathrm{Myr}$. The resulted total SFR $\left(1.45 \mathrm{M}_{\odot} \mathrm{yr}^{-1}\right.$, see Table 7) is about a factor of 2 higher than that of $\mathrm{Xu}$ et al. (1999), primarily due to the extinction correction.

Table 7. $\mathbf{H}_{\alpha}$ Luminosities and Star-formation Rate in SQ-A

\begin{tabular}{|c|c|c|c|c|}
\hline velocity components & $\begin{array}{c}\mathrm{f}_{H_{\alpha}} \\
\text { (no ext. corr.) } \\
10^{-14} \text { erg s}^{-1} \mathrm{~cm}^{-2}\end{array}$ & $\begin{array}{c}\mathrm{f}_{H_{\alpha}} \\
\text { (ext. corr.) } \\
10^{-14} \mathrm{erg} \mathrm{s}^{-1} \mathrm{~cm}^{-2}\end{array}$ & $\begin{array}{c}\mathrm{L}_{H_{\alpha}} \\
\text { (ext. corr.) } \\
10^{40} \text { erg s}^{-1}\end{array}$ & $\begin{array}{c}\text { S.F.R. } \\
\mathrm{M}_{\odot} \mathrm{yr}^{-1}\end{array}$ \\
\hline $6600 \mathrm{~km} \mathrm{~s}^{-1}$ & 8.31 & 52.4 & 40.6 & 1.25 \\
\hline $6000 \mathrm{~km} \mathrm{~s}^{-1}$ & 4.65 & 8.38 & 6.43 & 0.20 \\
\hline total & 13.0 & 60.8 & 47.0 & 1.45 \\
\hline
\end{tabular}

The star formation rate can also be estimated from the FIR luminosity. The $60 \mu m$ and $100 \mu m$ flux densities of SQ-A (Table 2) correspond to a FIR luminosity of $L_{r m 40--120 \mu m}=2.52 \times 10^{42} \mathrm{erg} \mathrm{s}^{-1}$. In order to obtain the total IR $(5-1000 \mu \mathrm{m})$ luminosity $L_{I R}$, we estimate the total emission of large grains using the median ratio of $L_{c d} / L_{40--120 \mu m}=2.06$ found by Popescu \& Tuffs (2002) for the cold dust emission in Im-BCD galaxies, and approximate the emission of small grains/large molecules by the $15 \mu \mathrm{m}$ luminosity $\left(\nu L_{\nu}\right)$ of SQ-A which is $1.82 \times 10^{42} \mathrm{erg} \mathrm{s}^{-1}$ (Xu et al. 1999). This results in $L_{I R}=7.01 \times 10^{42}$ $\mathrm{erg} \mathrm{s}^{-1}$. Then assuming 60 percent of the bolometric luminosity of the starburst is absorbed by dust and re-emitted in the IR (Xu et al. 1994), we obtain a total (bolometric) luminosity of the starburst of 1.17 $10^{43} \mathrm{erg} \mathrm{s}^{-1}$. Compared to the results of the same STARBURST simulation described above, the FIR luminosity corresponds to a star formation rate of $0.33 \mathrm{M}_{\odot} \mathrm{yr}^{-1}$. This is about a factor 4 lower than the star formation rate derived from the $\mathrm{H}_{\alpha}$ luminosities.

The discrepancy could be caused by the following factors:

(1) Possible over-correction of the $\mathrm{H}_{\alpha}$ extinction of the $6600 \mathrm{~km} \mathrm{~s}^{-1}$ component which is estimated using 
the Balmer decrement of M1. This could happen if M1, located at the core of the starburst, has encountered more extinction than HII regions elsewhere in SQ-A. However, this seems not the case because the Balmer decrement of source $\mathrm{Nd}$, which is at the periphery of the $6600 \mathrm{~km} \mathrm{~s}^{-1} \mathrm{H}_{\alpha}$ emission region, gives an $A_{H_{\alpha}}=1.9 \mathrm{mag}$, in good agreement with that of M1 $(2.0 \mathrm{mag})$. Furthermore, even without any extinction correction, the SFR estimated from the $\mathrm{H}_{\alpha}$ luminosity is already a factor of $\sim 2$ higher that that derived from the FIR luminosity. Therefore, the discrepancy is unlikely to be caused by over-correction of the $\mathrm{H}_{\alpha}$ extinction of the $6600 \mathrm{~km} \mathrm{~s}^{-1}$ component.

(2) Errors in the $f_{60 \mu m}$ and $f_{100 \mu m}$. A hint for this can be drawn from the relatively low $f_{60 \mu m} / f_{15 \mu m}$ ratio $(=5.5)$ of SQ-A, which is about a factor of 4 lower than the mean $(=21.5 \pm 9.4)$ of the eight closely interacting and starbursting galaxy pairs observed by Xu et al. (2000). This also explains why the star formation rate derived using $f_{60 \mu m}$ and $f_{100 \mu m}$ is significantly lower than that derived using $f_{15 \mu m}$ (Xu et al. 1999). Both $f_{60 \mu m}$ and $f_{100 \mu m}$ are derived after the subtraction of brighter sources NGC 7319 and NGC 7320, so could be seriously affected by errors in the subtraction.

(3) A top-heavy IMF. If the starburst has more massive ionizing stars than predicted by a Salpeter IMF, one will have more $\mathrm{H}_{\alpha}$ emission for a given bolometric luminosity. There has been evidence that starbursts may tend to have top-heavy IMFs (Rieke et al. 1980; Bernloehr 1993). Given the unusual nature of SQ-A (an IGM starburst triggered by a high speed collision), it will be very interesting to find out in future studies whether indeed it has an unusual IMF.

Future FIR observations with higher angular resolutions than ISOPHOT (e.g. SIRTF-MIPS observations) will distinguish the possibilities (2) and (3).

\section{2. $\quad$ Triggering Mechanism}

$\mathrm{Xu}$ et al. (1999) summarized the supporting evidence available by then for the argument that SQ-A is an ongoing starburst. These include (1) strong MIR emission, (2) high $\mathrm{H}_{\alpha}$ equivalent-line-width (>100A), (3) weak NIR (K band) emission. Dividing the stellar mass estimated from the K-band luminosity by the star-formation rate estimated from the MIR and $\mathrm{H}_{\alpha}$ luminosities, $\mathrm{Xu}$ et al. (1999) found the age of the starburst to be $\lesssim 10-20$ Myr. The " $\lesssim$ " sign reflects the possibility that some of the K-band emission is due to an underlying older stellar population (stars stripped from the member galaxies in previous galaxy-galaxy close-encounters). New HST observations of Gallagher et al. (2001) indeed found many very young $(\sim 5-6 \mathrm{Myr})$ star clusters in the region. Also, large amount of molecular gas $\left(510^{8}-10^{9} \mathrm{M}_{\odot}\right)$ has been discovered in this region (Gao \& Xu 2001; Smith \& Struck 2001; Lisenfeld et al. 2002), consistent with an ongoing starburst.

$\mathrm{Xu}$ et al. (1999) argued that this starburst is triggered directly by the collision between the intragroup cold gas (the $6600 \mathrm{~km} \mathrm{~s}^{-1}$ component of the HI) and the cold gas associated with the intruder galaxy NGC 7318b (the $6000 \mathrm{~km} \mathrm{~s}^{-1}$ component of the HI), and is not triggered by any tidal effects between NGC 7318b and other SQ members, so should not be treated as a 'tidal dwarf' as suggested by some other authors (e.g. Mendes de Oliveira et al. 2001). The supporting evidence for this argument includes:

(1) The $\mathrm{H}_{\alpha}$ data show that the star formation in SQ-A is occurring both in the IGM $\left(6600 \mathrm{~km} \mathrm{~s}^{-1}\right.$ component) and in the ISM of the intruder (the $6000 \mathrm{~km} \mathrm{~s}^{-1}$ component). In particular, the fact that the $\mathrm{H}_{\alpha}$ emission is dominated by the $6600 \mathrm{~km} \mathrm{~s}^{-1}$ component, conclusively rules out any interpretation for the starburst that involves only processes within the intruder NGC $7318 \mathrm{~b}$. 
(2) The age of the starburst ( $\sim 10 \mathrm{Myr})$ is consistent with the dynamical time scale of the high speed collision. The probability that both the starburst and the collision are happening simultaneously within such a short time scale would be very low if the former is not causally related to the latter.

(3) It is even more implausible that, in such a short time scale, two separated 'tidal dwarf' starbursts, one associated with the IGM (the $6600 \mathrm{~km} \mathrm{~s}^{-1}$ component) and the other with ISM of the intruder (the $6000 \mathrm{~km} \mathrm{~s}^{-1}$ component), are simultaneously happening (within such a small sky region) together with the collision. Therefore, the conclusion is almost inevitable that the three events (the collision, the two velocity components of the starburst) are directly related, as depicted in the collision induced starburst scenario (as opposite to the 'tidal dwarf' scenario).

Given the high collision velocity $\left(\sim 600 \mathrm{~km} \mathrm{~s}^{-1}\right)$ and the apparent link between SQ-A and the shock front in the $\mathrm{H}_{\alpha}$ map (Fig. 6), a candidate for the triggering mechanism of the starburst is the star formation induced by a shock, as originally modeled by Elmegreen \& Elmegreen (1978). Such a mechanism invokes the gravitational instability in the post-shock gas for the triggering of the star formation, and has been applied to star formation in the jet-induced emission line regions in the high-z radio galaxy $4 \mathrm{C} 41.17$ by Bicknell et al. (2000). However, in SQ-A, we did not detect a significant component in the $\mathrm{H}_{\alpha}$ emission associated with the post-shock gas, which should have redshift $\sim 6300 \mathrm{~km} \mathrm{~s}^{-1}$ (assuming that HI clouds in the $6000 \mathrm{~km} \mathrm{~s}^{-1}$ and the $6600 \mathrm{~km} \mathrm{~s}^{-1}$ systems have similar mass distributions). Instead, given that the two $\mathrm{H}_{\alpha}$ components are closely associated with the two $\mathrm{HI}$ velocity systems detected in this region (Williams et al. 2002), the star formation is apparently happening in the pre-shock gas (see Section 7.1 for arguments on why $6000 \mathrm{~km} \mathrm{~s}^{-1}$ gas is not post-shock gas).

A better model can be drawn from the theory developed by Jog \& Solomon (1992, hear after JS), initially aimed to explain the origin of the intense starbursts seen in colliding, gas-rich, field spiral galaxies. In that theory, it is assumed that the collisions between the HI clouds, which have much larger filling factors than the molecular clouds, of two colliding gas systems lead to the formation of a hot $(\sim 1 \mathrm{keV})$ ionized, high pressure remnant gas. The over-pressure due to this hot gas causes a radiative shock compression of the outer layers of pre-existing giant-molecular-clouds (GMCs), which are embedded in the HI clouds before the collision. The 'squeezing' radiative shock lasts only about $10^{4}$ years (the crossing time of individual HI clouds), too short to destroy a GMC but long enough to trigger instabilities in the thin outer layers. These layers become gravitationally unstable and a burst of massive star formation is ignited in the initially barely stable GMCs.

It is interesting to note that $\mathrm{Xu}$ et al. (1999) discounted this theory for the following considerations: (1) it was not clear whether there is any molecular gas in SQ-A region because it is very rare for molecular gas to be seen so far away ( $\gtrsim 20 \mathrm{kpc}$ ) from galaxy centers; and (2) according to Pietsch et al. (1997) SQ-A was in an X-ray hole in the ROSAT map, hence the signal for the hot remnant gas produced by the ongoing collision between HI clouds was missing. Since the publication of Xu et al. (1999), several new observations have shed new light on above issues, and lent support for the JS model. First of all, Gao \& Xu (2000) made the first detection of molecular gas in SQ-A with high angular resolution $(\sim 8$ ") interferometric CO observations using BIMA. This was later confirmed by single dish observations of Smith \& Stuck (2001) and Lisenfeld et al. (2002). The mass of the detected molecular gas is $\sim 10^{9} M_{\odot}$, and both velocity components (6600 and $6000 \mathrm{~km} \mathrm{~s}^{-1}$ ) were detected. Secondly, the new Chandra X-ray image of Trinchieri et al. (2003), which is much more sensitive than the ROSAT maps, shows that there is indeed an X-ray source at the position of the core of SQ-A (corresponding to the peak in the $\mathrm{H}_{\alpha}$ map of the $6600 \mathrm{~km} \mathrm{~s}^{-1}$ component and the peak in the radio continuum maps). 
Indeed it is expected in the scenario depicted by the JS model that the starburst should have two components with the same velocities as the colliding cold gas systems since the motions of the pre-existing GMCs (within which the starburst is taking place) are little affected by the collision. This is because (1) the GMCs do not collide with each other due to very low filling factors (Jog \& Solomon 1992); and (2) given GMCs' very high density and compact configuration, and the rather short time scale $\left(\sim 10^{4}\right.$ yrs $)$ for collisions between individual HI clouds (within which the GMCs are embedded), little momentum will be transferred from the GMCs to surrounding low density gas during the collision. The compression radiative shock by the remnant gas is symmetric, hence will not affact the momentum of the GMC's, either.

In this scenrio, the starburst starts immediately after the collision taking place. Following JS (see their Eq. 17), the star formation rate can be estimated as:

$$
S F R=1 M_{\odot} y r^{-1}\left(\frac{M_{m o l}}{10^{9} M_{\odot}}\right)\left(\frac{f}{0.2}\right)\left(\frac{\alpha_{m}}{0.1}\right)\left(\frac{S F E}{0.5}\right)\left(\frac{t}{10^{7} y r}\right)
$$

where $M_{\text {mol }} \sim 10^{9} M_{\odot}$ is the total molecular gas in this region, $f \sim 0.2$ is the fraction of this gas that is participating in the collision. It is conceivable that a large fraction of the two gas systems may miss the collision if they both are in arm-like configurations (when the collision is not perfect). $\alpha$ is another fraction, defined by the mean ratio of the mass of the unstable layer to the total mass of a GMC which is participating in the collision. JS found that typically $\alpha_{m}=0.1$. Following again JS, we assume that the typical star formation efficiency for the unstable GMC layer is $S F E=0.5$. The time for the two gaseous systems to pass each other is $t \sim 10^{7} \mathrm{yr}$. With these plausible parameters, the star formation rate derived in Eq. 7 agrees very well with what is observed for SQ-A (Table 7).

In principle, the triggering mechanism proposed for the starburst in SQ-A should also work in other regions in SQ where the large-scale collision is taking place. The condition is the availability of pre-existing GMCs. Indeed, in the south of NGC 7318b (around the position of RA(J2000) $=22^{h} 35^{m} 59^{s}$, $\left.\operatorname{Dec}(\mathrm{J} 2000)=33^{\circ} 57^{\prime} 30^{\prime \prime}\right)$, where marginally significant evidence for the $\mathrm{CO}$ emission could be found in the BIMA image (Gao \& Xu 2000), young star clusters ( $10^{7}$ yr, Gallagher et al. 2001) and the $\mathrm{H}_{\alpha}$ emission (Xu et al. 1999) indicate current star formation. This region was detected with bright X-ray emission (Pietsch et al. 1998; Sulentic et al. 2001; Trinchieri et al. 2003), and the long slit spectroscopic observations of Gallagher et al. (2001) show both the $5700 \mathrm{~km} \mathrm{~s}^{-1}$ and $6600 \mathrm{~km} \mathrm{~s}^{-1}$ systems. These are consistent with that the star formation in this region may also be triggered by the same mechanism as modeled by JS. Gao \& Xu (2000) did not detect any CO emission in the shock front. This may explain why there is no conspicuous star formation, either in the form of young clusters (Gallagher et al 2001) or as bright point sources in $\mathrm{H}_{\alpha}$ images (Xu e al. 1999), in this region. It should be noted that Lisenfeld et al. (2002) reported detection of the $\mathrm{CO}$ emission in the shock front region. However, given the large beam $\left(22^{\prime \prime}\right)$ of their single dish observations, it is not very certain that the emission is really from the shock front.

\section{Discussion}

\subsection{Is the $6000 \mathrm{~km} \mathrm{~s}^{-1}$ Component the Post-shock Gas?}

In this paper, we have accepted the suggestion of Moles et al. (1997) that both the $5700 \mathrm{~km} \mathrm{~s}^{-1}$ and $6000 \mathrm{~km} \mathrm{~s}^{-1} \mathrm{HI}$ gas components belong to NGC 7318b, the velocity difference due to the rotation. Williams et al. (2002) questioned such a scenario based on the apparent separation between the two HI components, in both spatial and velocity distributions. Sulentic et al. (2001) disputed this argument and 
further supported the suggestion of Moles et al. (1997). They pointed out the connection of the two HI components through the ionized gas in the shock front. Also, the Fabry-Pérot observations (Sulentic et al. 2001) demonstrate that the velocity of the emission line regions along an arc, which includes the shock front and links both the $5700 \mathrm{~km} \mathrm{~s}^{-1}$ and $6000 \mathrm{~km} \mathrm{~s}^{-1} \mathrm{HI}$ components, changes continuously from $\sim 5700 \mathrm{~km}$ $\mathrm{s}^{-1}$ to $\sim 6000 \mathrm{~km} \mathrm{~s}^{-1}$, indicating a kinematic connection between the two velocity systems.

An alternative picture, as suggested by Lisenfeld et al. (2002), is that the $6000 \mathrm{~km} \mathrm{~s}^{-1}$ gas is indeed linked to $5700 \mathrm{~km} \mathrm{~s}^{-1}$ component, but the velocity difference is due to the current interaction with the 6600 $\mathrm{km} \mathrm{s}^{-1}$ component, instead of the internal rotation of the ISM of NGC 7318b. Since the time scale is too short for any gravitational effects, the only way to accelerate the $5700 \mathrm{~km} \mathrm{~s}^{-1}$ gas to $6000 \mathrm{~km} \mathrm{~s}^{-1}$ during the current collision is through the shock. Hence, in sich a scenario, the $6000 \mathrm{~km} \mathrm{~s}^{-1}$ component should be the post-shock gas. However, our spectroscopic observations do not support this hypothesis. In both the SQ-A region and the shock front region, where there was no detection of the $5700 \mathrm{~km} \mathrm{~s}^{-1} \mathrm{HI}$ gas, there is no evidence for a $5700 \mathrm{~km} \mathrm{~s}^{-1}$ component in the ionized gas, either. If all the $6000 \mathrm{~km} \mathrm{~s}^{-1}$ cold gas (HI, molecular, and the ionized) were processed gas already passed through the shock front, then there would be nothing left for the $6600 \mathrm{~km} \mathrm{~s}^{-1}$ gas to collide with. Our conclusion is that the $6000 \mathrm{~km} \mathrm{~s}^{-1}$ component is not the post-shock gas.

\subsection{Distribution of Pre-shock Gas}

Nevertheless, the $5700 \mathrm{~km} \mathrm{~s}^{-1}$ and $6000 \mathrm{~km} \mathrm{~s}^{-1}$ components of the HI gas do show peculiarities: (1) they are outside the main body of NGC 7318b; (2) their maps do not show a rotating-disk morphology, even after adding the ionized gas in the shock front; (3) in particular, the $6000 \mathrm{~km} \mathrm{~s}^{-1}$ component appears to be a round, extended cloud centered at SQ-A (Fig. 9 of Williams et al. 2002), with little sign of any substructure.

Moles et al. (1997) and Sulentic et al. (2001) argued that NGC 7318b had been a 'normal' gas-rich galaxy before it entered SQ. In this scenario, it is difficult to explain the above peculiarities of the HI gas that is presumably associated with the galaxy. There are also 'abnormal' features in its optical morphology: (1) its outer optical disk is one-side loped toward the north; (2) it has several long, open arms which are usually seen in interacting galaxies (the so called 'tidal tails'). Williams et al. (2002) argued that NGC 7318b is perhaps not entering the SQ the first time. However, its relative velocity is too high for it to be gravitationally bound to the SQ system, and therefore repeat passages seem to be unlikely.

Another possibility is that these abnormal features are due to interaction with the elliptical galaxy NGC 7318a, which has to be at least $\sim 100 \mathrm{kpc}$ behind NGC 7318b at the moment, although the projected distance is only $\sim 10 \mathrm{kpc}$. The requirement on the large line-of-sight distance between NGC 7318a and NGC $7318 \mathrm{~b}$ is because the time scale for tidal effects is a few $100 \mathrm{Myr}$. Within this time, with the projected relative velocity $\left(\delta V \simeq 900 \mathrm{~km} \mathrm{~s}^{-1}\right)$, NGC $7318 \mathrm{~b}$ would have moved a few $100 \mathrm{kpc}$ away from NGC 7318a since the close encounter. This also requires that NGC 7318b moves almost along the line of the sight, otherwise the projected distance between NGC 7318a and NGC 7318b would be much larger. Indeed, from the narrow width of the shock front, and from the fact that in the shock front and in SQ-A, emission line systems with velocities of $\sim 6600 \mathrm{~km} \mathrm{~s}^{-1}$ and of $\sim 6000 \mathrm{~km} \mathrm{~s}^{-1}$ are found on top of each other, Sulentic et al. (2001) argued that the direction of the relative velocity of NGC 7318b must be close to the line of sight. Future works such as higher sensitivity and higher resolution HI maps and detailed theoretical simulations will help to solve the puzzle related to the HI and optical morphology of NGC 7318b. 
As shown by Sulentic et al. (2001), the pre-shock $6600 \mathrm{~km} \mathrm{~s}^{-1}$ cold gas is in a huge arc (longer than $100 \mathrm{kpc}$ ), with the SQ-A in the northwest tip. This gaseous arc is likely to be a tidal feature related to a previous close encounter between member galaxies, which could have happened a few 100 Myr ago (Moles et al. 1997; Sulentic et al. 2001). The concentration of the HI gas (Williams et al. 2002) and the molecular gas (Gao \& Xu 2000) in SQ-A, which is more than $20 \mathrm{kpc}$ away from any galaxy center, may indeed have the similar origin as those HI-knots observed along the tidal tails (particularly at tips of tidal tails) of interacting galaxies (Hibbard \& von Gorkom 1996; Duc et al. 2000; Braine et al. 2001), although the triggering mechanism of the IGM starburst is different from those of star-forming 'tidal dwarfs' as modeled by Barnes, J.E. \& Hernquist (1992) and Elmegreen et al. (1993).

\section{Conclusions}

The compact galaxy group Stephan's Quintet (SQ) provides a unique target in the local universe for studying the effects of high velocity collisions $\left(\sim 1000 \mathrm{~km} \mathrm{~s}^{-1}\right)$ between two systems rich in cold gas. The two phenomenal events currently taking place in the IGM of SQ, namely the large scale shock $(\sim 40 \mathrm{kpc})$ and the IGM starburst $\mathrm{SQ}-\mathrm{A}\left(\mathrm{SFR}=1.45 \mathrm{M}_{\odot} \mathrm{yr}^{-1}\right)$, are very likely to be triggered by the same ongoing collision between the intruder galaxy NGC $7318 \mathrm{~b}\left(v=5700 \mathrm{~km} \mathrm{~s}^{-1}\right)$ and the IGM $\left(v=6600 \mathrm{~km} \mathrm{~s}^{-1}\right)$. In this paper, we provided new constraints on the physical conditions in the IGM involved, and investigated the physical mechanism linking these events with the collision using new far infrared (FIR) images at $60 \mu m$ and $100 \mu \mathrm{m}$ (ISOPHOT C-100 camera), radio continuum images at $1.4 \mathrm{GHz}$ (VLA B-array) and $4.86 \mathrm{GHz}$ (VLA C-array), and long-slit optical spectrographs (Palomar 200" telescope).

We found that the shock front, which appears as a radio ridge and dominates the radio continuum emission of SQ, has a steep nonthermal spectral index $(\alpha=0.93 \pm 0.13)$. Its FIR-to-radio flux ratio is extremely low $(q<0.59)$ compared to that of galaxies $(<q>=2.3 \pm 0.2)$, consistent with the hypotheses that the relativistic electrons responsible for the radio emission are accelerated by the large scale shock (in contrast to the relativistic electrons in galaxy disks which are likely to be accelerated by SNRs). Its observed IR emission can be explained in terms of collisional heating of dust grains by the plasma immediately downstream of the shock. The long-slit spectra of sources in this region have typical emission line ratios of shock-excited gas. The very broad line line widths (FWHM $\geq 1000 \mathrm{~km} \mathrm{~s}^{-1}$ ), and the fact that in some cases more than two velocity systems were detected along the same line of sight, provide further evidence for an ongoing collision in this region. The magnetic field strength estimated using the minimum-energy assumption is $\approx 10 \mu \mathrm{G}$. The implied energy density of the electro-magnetic field is significantly lower than the IGM thermal energy density derived from the X-ray emission, indicating a minor role played by the electro-magnetic force on the dynamics in the shock front. No linearly polarized emission brighter than $50 \mu \mathrm{Jy}$ beam $^{-1}$ was found in any component of SQ at either 1.40 or $4.86 \mathrm{GHz}$, indicating that the magnetic fields may be disordered, and both "beam depolarization" and Faraday rotation may have caused the reduced polarization.

The IGM starburst SQ-A was clearly detected in both the FIR and the radio bands. Its radio spectral index $(\alpha=0.8 \pm 0.3)$ and the FIR-to-radio ratio $(q=2.0 \pm 0.4)$ are consistent with those of star formation regions. The optical spectra of two sources in this region, M1 $\left(v=6600 \mathrm{~km} \mathrm{~s}^{-1}\right)$ and M2 $\left(v=6000 \mathrm{~km} \mathrm{~s}^{-1}\right)$, have typical line ratios of HII regions. The metallicity of M1 is $12+\log [O / H]=8.76 \pm 0.06$ and that of M2 is $12+\log [O / H]=8.95 \pm 0.09$, both being slightly higher than the solar value $(12+\log [O / H]=8.69 \pm 0.05$, Allende Prieto et al. 2001). This result confirms that the IGM is stripped gas from galaxies and rules out the possibility that it is primordial. According to the Balmer decrement of M1, the $6600 \mathrm{~km} \mathrm{~s}^{-1}$ component 
of the IGM starburst is heavily obscured $\left(A_{\alpha}=2.0\right)$, while the Balmer decrement of M2 indicates a moderate extinction $\left(A_{\alpha}=0.64\right)$ for the $6000 \mathrm{~km} \mathrm{~s}^{-1}$ component. The star formation rate estimated from the extinction-corrected $\mathrm{H}_{\alpha}$ luminosity of SQ-A is $1.45 \mathrm{M}_{\odot} \mathrm{yr}^{-1}$, of which $1.25 \mathrm{M}_{\odot} \mathrm{yr}^{-1}$ due to the 6600 $\mathrm{km} \mathrm{s}^{-1}$ component and $0.20 \mathrm{M}_{\odot} \mathrm{yr}^{-1}$ due to the $6000 \mathrm{~km} \mathrm{~s}^{-1}$ component. The SFR estimated using the FIR luminosity is significantly lower $\left(\mathrm{SFR}=0.33 \mathrm{M}_{\odot}\right)$. The discrepancy is due either to errors in the FIR flux densities or to a top-heavy IMF. The very good agreement in velocity between $\mathrm{H}_{\alpha}$ and $\mathrm{HI}$ (Williams et al. 2002) for both components suggests strongly that the starburst is occurring in the pre-shock gas rather than in the post-shock gas. A model (Jog \& Solomon 1992) based on a scenario in which a starburst is triggered in the outer layers of pre-existing (pre-shock) GMCs compressed by surrounding shocked gas (which is the HI gas before the collision), can be applied to SQ-A and explain the observed SFR, the two components of the starburst, and the apparent link between SQ-A and the large scale shock front.

This research has made use of the NASA/IPAC Extragalactic Database (NED) which is operated by the Jet Propulsion Laboratory, California Institute of Technology, under contract with the National Aeronautics and Space Administration. We thank Cristina Popescu and Jack Sulentic for helpful discussions.

\section{REFERENCES}

Allam, S., Assendorp, R., Longo, G., Braun, M., Richter G.: 1996, A\&AS, 117, 39.

Allen, R.J., Hartsuiker, J.W. 1972, Nature, 239, 324.

Allende Prieto, C., Lambert, D.L., Asplund, M. 2001, ApJ, 556, 63.

Aoki, K., Kosugi, G., Wilson, A.S., Yoshida, M. 1999, ApJ, 521, 565

Baars, J.W.M., Genzel, R., Pauliny-Toth, I.I.K., Witzel, A. 1977, A\&A, 61, 99

Barnes, J., Hernquist, L. 1992, Nature, 360, 715.

Bernloehr, K. 1993, A\&A, 268, 25.

Bicknell, G.V., Sutherland, R.S., van Breugel, W.J.M., Dopita, M.A., Dey, A., Miley, G.K. 2000, ApJ, 540, 678.

Braine, J., Duc, P.-A., Lisenfeld, U., Charmandaris, V., Vallejo, O., Leon, S., Brinks, E. 2001, A\&A, 378, 51.

Condon, J.J. 1992, ARAA, 30, 575.

Condon, J.J., Broderick, J.J. 1988, AJ, 96, 30.

De Jong, T. Klein, U., Wielebinski, R., Wunderlich, E. 1985, A\&A 147, L6.

Duc, P.-A., Brinks, E., Springel, V., Pichardo, B., Weilbacher, P.; Mirabel, I.F. 2000, AJ, 120, 1238.

Dopita, M.A., Sutherland, R. 1995, ApJ, 455, 468.

Dopita, M.A., Sutherland, R. 1996, ApJS, 102, 161.

Draine, B.T., Lee, H.M. 1984, ApJ, 285, 89.

Draine, B.T., Salpeter, E.E. 1979, ApJ, 231, 77.

Dwek, E., Foster, S.M., Vancura, O. 1996, ApJ, 457, 244. 
Dwek, E., Werner, M. W. 1981, ApJ, 248, 138.

Elmegreen, B.G., Elmegreen, D.M. 1978, ApJ, 220, 1051.

Elmegreen, B.G., Kaufman, M., Thomasson, M. 1993, ApJ, 412, 90.

Evans, I.N., Dopita, M.A. 1985, ApJS, 58, 125.

Gao, Y., Xu, C. 2000, ApJL, 542, L83.

Gallagher, S., Charlton, J.S., Hunsberger, S.D., Zaritsky, D., Whitmore, B.C. 2001, AJ, 122, 163.

Heckman, T.M., Baum, S.A., van Breugel, W.J.M., McCarthy, P. 1989, ApJ, 338, 48.

Helou, G.X., Soifer, B.T., Rowan-Robinson, M. 1985, ApJL, 298, L7.

Hibbard, J.E., Von Gorkom, J.H. 1996, AJ, 111, 655.

Hoernes, P., Berkhuijsen, E.M., Xu, C. 1998, A\&A, 334, 57.

Jog, C.J. \& Solomon, P.M. 1992, ApJ, 387, 152.

Kenney, J.D.P., Rubin, V.C., Planesas, P., Young, J.S. 1995, ApJ, 438, 549.

Kessler, M.F., Steinz, J.A., Anderegg, M.E. et al. 1996, A\&A, 315, L27.

Kewley, L.J., Dopita, M.A., 2002, ApJS, 142, 35.

Klemola, A.R., Jones, B.F., \& Hanson, R.B. 1987, AJ, 94, 501

Leitherer, C., Schaerer, D., Goldader, J.D., Delgado, R.M.G., Robert, C., Kune, D.F., de Mello, D.F., Devost, D., Heckman, T.M. 1999, ApJS, 123, 3.

Lisenfeld, U., Braine, J., Duc, P.-A., Leon, S., Charmandaris, V., Brinks, E. 2002, A\&A, 394, 823.

Mendes de Oliveira, C., Plana, H., Amram, P., Balkowski, C., Bolte, M. 2001, AJ, 121, 2524.

Moles, M., Márquez, I. Sulentic, J.W. 1998, A\&A, 243.

Moles, M., Sulentic, J.W., Márquez, I. 1997, ApJ, 485, 69.

Ohyama, Y., Nishiura, S., Murayama, T. Yaniguchi, Y. 1998, ApJL, 492, L25.

Oke, J. B. 1990, AJ, 99, 1621.

Plana, H., Mendes de Oliveira, C., Amram, P., Bolte, M., Balkowski, C., Boulesteix, J. 1999, ApJ, 516, L69.

Pietsch, W., Trinchieri, G., Arp, H., Sulentic, J.W. 1997, A\&A, 322, 89.

Popescu, C.C. Tuffs, R.J. 2002, MNRAS 335, L41.

Popescu, C.C., Tuffs, R.J., Völk, H.J., Pierini, D., Madore, B.F. 2002, ApJ, 567. 221.

Popescu, C.C, Tuffs, R.J., Fischera, J., Völk, H.J., 2000, A\&A, 354, 480.

Rieke, G.H., Lebofsky, M.J., Thompson, R.I., Low, F.J. \& Tokunaga, A.T. 1980 ApJ, 238, 24.

Saracco, P., Ciliegi, P. 1995, A\&A, 301, 348.

Savage, B.D., Mathis, J.S. 1979, ARAA, 17, 73.

Shostak, G.S., Sullivan III, W.T., Allen, R.J. 1984, A\&A, 139, 15.

Smith, B., Struck, C. 2001, AJ, 121, 710.

Stephan, M. E. 1877, C. R. Acad. Sci. Paris, 84, 641.

Sulentic, J.W., Pietsch, W., Arp, H. 1995, A\&A, 298, 420. 
Sulentic, J.W., Rosado, M., Dultzin-Hacyan, D., Verdes-Montenegro, L., Trinchieri, G., Xu. C., Pietsch, W. 2001, AJ, 122, 2993.

Trinchieri, G., Sulentic, J.W., Breitschwerdt, D., Pietsch, W. 2003, A\&A, 401, 173.

Tuffs, R.J., Gabriel, C. 2003, A\&A, in press.

Tuffs, R.J., Popescu, C.C. 2002, in proc. "Exploiting the ISO Data Archive. Infrared Astronomy in the Internet Age", Siguenza, Spain 24-27 June, 2002, to be pulished in ESA Special Publication series (SP-511).

Tuffs, R.J., Popescu, C.C., Pierini, D., Völk, H.J., Hippelein, H., Leech, K., Metcalfe, L., Heinrichsen, I., Xu, C., 2002a, ApJS, 139, 37.

Tuffs, R.J., Popescu, C.C., Pierini, D., Völk, H.J., Hippelein, H., Leech, K., Metcalfe, L., Heinrichsen, I., $\mathrm{Xu}, \mathrm{C} ., 2002 \mathrm{~b}$, ApJS, 140, 609.

van der Hulst, J.M., Rots, A.H. 1981, AJ, 86, 1775.

Völk, H., Xu, C. 1994, Infrared Phys. Technol. 35, 527.

Yun, M.S., Verdes-Montenegro, L., del Olmo, A. \& Perea, J. 1997, ApJ, 475, 21.

Williams, B.A., Yun, M.S., Verdes-Montenegro, L. 2002, AJ, 123, 2417.

Xanthopoulos, E., Muxlow, T.W.B., Thomasson, P., Garrington, S.T. 2002, preprint (astro-ph/0202332).

Xu, C., Gao, Y., Mazzarella, J., Lu, N.Y., Sulentic, J.W., Domingue, D.L. 2000, ApJ. 541, 644.

Xu, C., Klein , U., Meinert, D., Wielebinski, R., Haynes, R.F. 1992, A\&A, 257.

Xu, C., Lisenfeld, U., Völk, H.J., Wunderlich, E. 1994, A\&A, 282, 19.

Xu, C., Sulentic, J.W., Tuffs, R. 1999, ApJ, 512, 178. 
This figure "fig1a.gif" is available in "gif" format from: http://arxiv.org/ps/astro-ph/0306261v1 
This figure "fig1b.gif" is available in "gif" format from: http://arxiv.org/ps/astro-ph/0306261v1 
This figure "fig2a.gif" is available in "gif" format from: http://arxiv.org/ps/astro-ph/0306261v1 
This figure "fig2b.gif" is available in "gif" format from: http://arxiv.org/ps/astro-ph/0306261v1 
This figure "fig2c.gif" is available in "gif" format from: http://arxiv.org/ps/astro-ph/0306261v1 
This figure "fig2d.gif" is available in "gif" format from: http://arxiv.org/ps/astro-ph/0306261v1 
This figure "fig3a.gif" is available in "gif" format from: http://arxiv.org/ps/astro-ph/0306261v1 
This figure "fig3b.gif" is available in "gif" format from: http://arxiv.org/ps/astro-ph/0306261v1 
This figure "fig4a.jpg" is available in "jpg" format from: http://arxiv.org/ps/astro-ph/0306261v1 
This figure "fig4b.jpg" is available in "jpg" format from: http://arxiv.org/ps/astro-ph/0306261v1 
This figure "fig5.gif" is available in "gif" format from: http://arxiv.org/ps/astro-ph/0306261v1 
This figure "fig6.gif" is available in "gif" format from: http://arxiv.org/ps/astro-ph/0306261v1 
This figure "fig7.gif" is available in "gif" format from: http://arxiv.org/ps/astro-ph/0306261v1 
This figure "fig8.gif" is available in "gif" format from: http://arxiv.org/ps/astro-ph/0306261v1 
This figure "fig9.gif" is available in "gif" format from: http://arxiv.org/ps/astro-ph/0306261v1 
This figure "fig10.gif" is available in "gif" format from: http://arxiv.org/ps/astro-ph/0306261v1 
This figure "fig11.gif" is available in "gif" format from: http://arxiv.org/ps/astro-ph/0306261v1 